\title{
Nanocompositional Electron Microscopic Analysis and Role of Grain Boundary Phase of Isotropically Oriented Nd-Fe-B Magnets
}

\author{
Gregor A. Zickler and Josef Fidler \\ Institute of Solid State Physics, TU Wien, Wiedner Hauptstrasse 8-10, 1040 Vienna, Austria \\ Correspondence should be addressed to Josef Fidler; josef.fidler@tuwien.ac.at
}

Received 6 February 2017; Accepted 13 March 2017; Published 6 April 2017

Academic Editor: Jamal Berakdar

Copyright (C) 2017 Gregor A. Zickler and Josef Fidler. This is an open access article distributed under the Creative Commons Attribution License, which permits unrestricted use, distribution, and reproduction in any medium, provided the original work is properly cited.

\begin{abstract}
Nanoanalytical TEM characterization in combination with finite element micromagnetic modelling clarifies the impact of the grain misalignment and grain boundary nanocomposition on the coercive field and gives guidelines how to improve coercivity in NdFe-B based magnets. The nanoprobe electron energy loss spectroscopy measurements obtained an asymmetric composition profile of the Fe-content across the grain boundary phase in isotropically oriented melt-spun magnets and showed an enrichment of iron up to 60 at $\%$ in the $\mathrm{Nd}$-containing grain boundaries close to $\mathrm{Nd}_{2} \mathrm{Fe}_{14} \mathrm{~B}$ grain surfaces parallel to the $c$-axis and a reduced iron content up to $35 \%$ close to grain surfaces perpendicular to the $c$-axis. The numerical micromagnetic simulations on isotropically oriented magnets using realistic model structures from the TEM results reveal a complex magnetization reversal starting at the grain boundary phase and show that the coercive field increases compared to directly coupled grains with no grain boundary phase independently of the grain boundary thickness. This behaviour is contrary to the one in aligned anisotropic magnets, where the coercive field decreases compared to directly coupled grains with an increasing grain boundary thickness, if $J_{s}$ value is $>0.2 \mathrm{~T}$, and the magnetization reversal and expansion of reversed magnetic domains primarily start as Bloch domain wall at grain boundaries at the prismatic planes parallel to the $c$-axis and secondly as Néel domain wall at the basal planes perpendicular to the $c$-axis. In summary our study shows an increase of coercive field in isotropically oriented Nd-Fe-B magnets for GB layer thickness $>5 \mathrm{~nm}$ and an average $\left\langle J_{s}\right\rangle$ value of the GB layer $<0.8 \mathrm{~T}$ compared to the magnet with perfectly aligned grains.
\end{abstract}

\section{Introduction}

The increasing demand of high-performance rare earth permanent magnets with a high coercive field and an energy density product value suitable for large scale applications in wind turbines and electrically powered automotive devices led to the development of heavy rare earth lean/rare earthfree Nd-Fe-B based magnets and to the optimization of the complex multiphase microstructure of the magnets [1]. The hard magnetic properties are primarily controlled by the size, shape, and misalignment of the hard magnetic grains and their distributions and secondarily by the occurrence of other nonmagnetic and soft magnetic phases [2-4]. In addition, the coercive field also strongly depends on the intergranular grain boundary (GB) phases separating the hard magnetic grains $[5,6]$. The role of dopant elements, the thickness, and magnetic properties of the GB-phases have extensively been studied during the last 30 years [7, 8]. Local changes of the exchange coupling between grains and the decrease of the anisotropy field and demagnetizing field at/near intergranular phases considerably reduce the overall coercive field. First principles ab initio calculations claimed that even an antiparallel exchange coupling between a crystalline $\alpha$-Fe phase and the prismatic $\{100\}$ planes of $\mathrm{Nd}_{2} \mathrm{Fe}_{14} \mathrm{~B}$ would be energetically favorable, while a positive exchange-coupling constant was predicted in the $\mathrm{Nd}_{2} \mathrm{Fe}_{14} \mathrm{~B}$ $(001) / \alpha$-Fe interface [9].

Advances in electron microscopic characterization technology have greatly improved the ability to quantify real microstructures found in Nd-Fe-B magnets. These techniques, in combination with finite element micromagnetic modelling, are improving the understanding of magnetization 
reversal processes and coercivity mechanisms. Micromagnetic simulations give a deep insight into the mechanisms that cause magnetization reversal at external fields well below the anisotropy field [10]. Nowadays, the new nanoanalytical electron microscopic techniques with atomic resolution allow the creation of precise microstructural models suitable for the numerical micromagnetic calculation of the demagnetization curve including the coercive field value. A recent high resolution TEM/STEM investigation of the intergranular GB-phase of a large grained, anisotropic sintered heavy rare earth-free $\mathrm{Nd}-\mathrm{Fe}-\mathrm{B}$ magnet with grain sizes up to several microns revealed a difference in composition for grain boundaries parallel (large Fe-content) and perpendicular (low Fe-content) to the alignment direction [11]. This combined TEM/STEM and micromagnetic study of the anisotropic nature of grain boundaries shows a decrease of the coercive field with an increasing thickness of the grain boundary layer.

Two quite distinct methods are in commercial use for producing Nd-Fe-B magnets: the rapid-solidification technique of melt spinning and the traditional powder-metallurgysintering approach. The present study compares different microstructures of various melt-spun materials with isotropically oriented hard magnetic grains with a grain size ranging from $20 \mathrm{~nm}$ to $100 \mathrm{~nm}$. The melt-spinning procedure involves the ejection of a molten starting alloy through a crucible orifice onto the surface of a substrate copper disc with a high rotating speed [12]. The microstructure and magnetic properties of melt-spun neodymium-iron-boron ribbons are sensitively dependent on the quench rate. The resulting hysteretic properties of an individual magnet material strongly depend on their nominal composition, microstructure, and processing parameters [13]. Melt-spun magnet materials have widely been used for bonded and hot deformed type magnets so far. Hot-pressed melt-spun nanocrystalline heavy rare earth-free $\mathrm{Nd}-\mathrm{Fe}-\mathrm{B}$ magnets are promising candidates for a low cost solution for applications that require thermal stability up to $175^{\circ} \mathrm{C}-200^{\circ} \mathrm{C}$ [14].

The aim of the present paper is to determine the influence of the grain size, orientation of grains, and nanocomposition of GBs on the coercive field and magnetization reversal behaviour by a combined TEM/STEM and micromagnetic study with special emphasis on the nanoanalytical, high resolution EELS characterization of isotropically oriented GBs. The microstructural model structure based on an anisotropic compositional behaviour of GBs parallel and perpendicular to the easy axis of the grains which is used for the numerical micromagnetic simulations has been derived from the detailed nanoanalytical TEM/STEM analysis.

\section{Materials}

In the present study we investigated the microstructure of three rapidly quenched $\mathrm{Nd}-\mathrm{Fe}-\mathrm{B}$ ribbons in a nanoanalytical TEM/STEM study, which were provided by Magnequench Technology Center, Singapore. The isotropic RErich two-phase ribbon (MQU-F) with the nominal chemical composition (Pr,Nd) ${ }_{13.6} \mathrm{Fe}_{73.6} \mathrm{Co}_{6.6} \mathrm{Ga}_{0.6} \mathrm{~B}_{5.6}$ [15] has a distinct $3 \mathrm{~nm}-6 \mathrm{~nm}$ thick RE-rich GB-phase separating the isotropically oriented equiaxed and platelet shaped $\mathrm{Nd}-\mathrm{Fe}-$ $\mathrm{B}$ grains. The isotropic fine grained ribbon (MQP-B+) with the nominal chemical composition $\mathrm{Nd}_{12.4} \mathrm{Fe}_{77.3} \mathrm{Co}_{5.2} \mathrm{~B}_{5.2}$ [16] is enriched in " $\mathrm{Fe}+\mathrm{Co}$ " and possesses therefore a $1 \mathrm{~nm}-3 \mathrm{~nm}$ thin "Fe + Co"-rich GB-phase separating the isotropically orientated equiaxed $\mathrm{Nd}-\mathrm{Fe}-\mathrm{B}$ grains. In comparison an isotropically oriented and large grained nanocomposite with additional soft magnetic $\alpha$-Fe and $\mathrm{Nb}$-granular phases and without a GB-phase between the hard magnetic grains has been investigated.

\section{Methods}

The nanoanalytical and structural investigations of the rapidly quenched $\mathrm{Nd}-\mathrm{Fe}-\mathrm{B}$ permanent magnet materials have been carried out with an analytical field emission transmission electron microscope (TEM) (FEI Tecnai F20) at $200 \mathrm{kV}$, which is equipped with a silicon drift energy dispersive X-ray (EDX) detector, a Gatan GIF Tridiem image filter and electron energy loss spectrometer (EELS) and a high angle annular dark field (HAADF) detector. Conventional sample TEM preparation including cutting, polishing, and ion milling in a Precision Ion Polishing System (PIPS) from Gatan was conducted. The structural investigations were performed with Fast Fourier Transformation (FFT) of high resolution TEM/STEM (HRTEM) images and selected area electron diffraction (SAED).

EELS experiments were conducted to accurately determine the relative chemical composition of the intergranular phases via the $k$-factor method. This method calculates the relative atomic percentage of an element (e.g., $\mathrm{Nd}$ ) with respect to another element (e.g., Fe) from the ratio of their edge intensities in the EELS (or EDX) spectrum via the $k$-factor (e.g., $k(\mathrm{Nd} / \mathrm{Fe})$ ), which was derived from the measurement of a standard specimen (e.g., $\mathrm{Nd}_{2} \mathrm{Fe}_{14} \mathrm{~B}$ single crystal). TEM specimens with a relative thickness $t / \lambda<0.7$, where $t$ is the absolute specimen thickness and $\lambda$ the mean free path in the specimen, were used in these experiments. Firstly, the $k$-factors of $\mathrm{Pr} / \mathrm{Fe}$ and $\mathrm{Nd} / \mathrm{Fe}$ were calculated from EELS spectra of single crystalline $\operatorname{Pr}_{2} \mathrm{Fe}_{14} \mathrm{~B}$ and $\mathrm{Nd}_{2} \mathrm{Fe}_{14} \mathrm{~B}$ standards. Secondly, the background in the EELS spectra was fitted with a power-law function and subtracted, which resulted in the edge intensities of the elements. Thirdly, the relative atomic composition was calculated from the edge intensities via the $k$-factors. The determination of the relative chemical composition via the $k$-factor method is accurate for $t / \lambda<1.0$ with a relative error of $\pm 5 \%$ [17]. An optimized background model was used to measure the $\mathrm{Fe}-\mathrm{L}_{2,3}$ ionization edge due to its close vicinity to the F-K edge and the $\mathrm{Nd}-\mathrm{M}_{4,5}$ ionization edge due to its close vicinity to the $\operatorname{Pr}-\mathrm{M}_{4,5}$ edge [18]. To avoid the development of an oxidized layer on the surface of the TEM specimen, precise precautions were taken. The influence of the electron beam broadening and the tilt of the GBs with respect to the incident electron beam on the chemical composition of $2 \mathrm{~nm}-6 \mathrm{~nm}$ thin GBs, as described in our previous publication [11], were taken into account. The higher yield in the elastic scattering events in EELS with respect to EDX [19] leads to a shorter acquisition time of each spectrum in a line scan. This is an advantage especially 


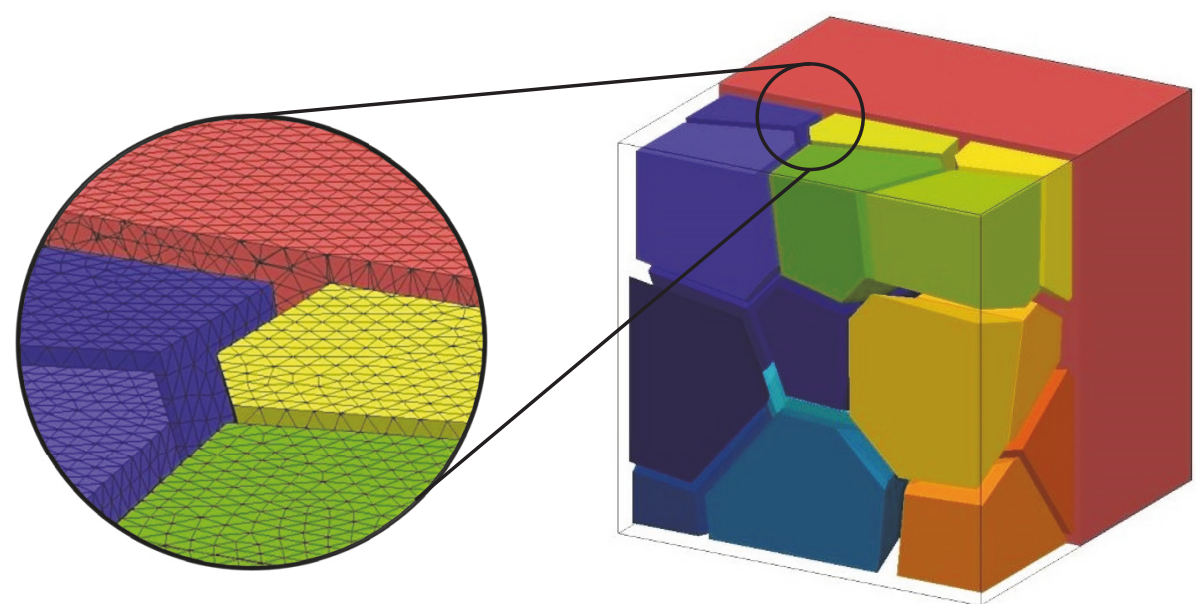

FIGURE 1: Micromagnetic finite element model structure with 29 Voronoi grains separated by a GB-phase with a thickness of about $10 \%$ of the grain diameter.

in the chemical analysis of thin GBs in thin $(<50 \mathrm{~nm})$ TEM specimens.

The finite element software package FEMME, which is a hybrid finite element/boundary element method code, was used for the numerical micromagnetic simulations [20]. On each point of the finite element mesh the Landau-LifshitzGilbert equation is being solved [21]. Besides the intrinsic magnetic properties, namely, the exchange constant $A$, the saturation polarization $J_{s}$, and the uniaxial magnetocrystalline anisotropy constant $K_{1}$, also the direction of the easy axis (direction of $K_{1}$ ) of a volume of a phase, which can be set with the polar angle $\vartheta$ and the azimuthal angle $\varphi$, is an input parameter for the simulation. $K_{1}$ was set to zero in the GBs, since it is expected to have a negligibly small or zero value. The long range demagnetizing field and the direct exchange coupling between neighbouring atomic moments in the hard magnetic grains and soft magnetic grain boundary layers strongly influence the magnetization reversal. Besides the exchange and the demagnetizing field, the magnetocrystalline anisotropy and the misorientation of the individual grains also contribute to the resulting magnetization reversal and coercivity [10].

Realistic finite element granular structures based on TEM investigations of melt-spun $\mathrm{Nd}-\mathrm{Fe}-\mathrm{B}$ magnets have been generated using the Voronoi algorithm [22]. This algorithm creates a unique volume decomposition based on a set of seeding points, similar to the Wigner-Seitz cell construction. We used the voro++ code [23] to create a Voronoi structure of equiaxed grains. The output from voro++ acts as an input for a Salome [24] script that creates a finite element discretization (mesh) of the granular structure. Two finite element model structures were created, one with directly coupled grains and one with a grain boundary phase with an approximate thickness of $10 \%$ of the grain size (Figures 1 and 2). The distribution of the easy axes of an isotropically orientated magnet is equal to the random distribution of points on a half sphere with a calculated azimuthal angle $\varphi=2 \pi \cdot u$ and polar angle $\vartheta=\cos ^{-1}(v)$, where $u$ and $v$ have to be chosen from random variates between 0 and 1 . This results in an average misorientation angle $\left\langle\vartheta_{0}\right\rangle=60^{\circ}$ and a projection of the magnetization parallel to the external field of $0.5 J_{s}$ $[25,26]$.

For a clear distinction between GBs parallel and perpendicular to the external field and the $c$-axis of the adjacent grains a simple two-grain model structure with an edge length of $40 \mathrm{~nm}$ was created and meshed with the software package GID version 12.0.4 [27] (Figure 3). Two $\mathrm{Nd}_{2} \mathrm{Fe}_{14} \mathrm{~B}$ grains are separated by a GB-phase consisting of two equally thick GB-volumes with a total GB thickness between 2, 4, 5,6 , and $8 \mathrm{~nm}$. All model structures were discretized with a $0.5 \mathrm{~nm}-2.5 \mathrm{~nm}$ mesh size, where the mesh tessellation was chosen in a way to ensure that the smallest GB volume has at least one central node surrounded with the nearest neighbours corresponding to GB material.

\section{Results and Discussion}

\subsection{TEM/STEM Characterization and Micromagnetic Simulation}

4.1.1. Isotropic RE-Rich Two-Phase Melt-Spun Ribbon (MQU$F)$. The polycrystalline microstructure of a rapidly quenched MQU-F ribbon with isotropic orientated $c$-axis of hard magnetic $\mathrm{Nd}-\mathrm{Fe}-\mathrm{B}$ grains with a size ranging from $20 \mathrm{~nm}$ to over $100 \mathrm{~nm}$ is shown in the TEM bright field (BF) and HAADF images of Figure 4. The contrast of the TEM-BF image is originated by the combination of orientation/diffraction contrast and absorption contrast, which depends on the thickness and average density of the TEM specimen leading to the bright contrast of the GB-phase. A HAADF image is generated in the STEM mode and the origin of the images contrast depends on the chosen camera length. At a cameral length $(\mathrm{cl})$ below $\approx 80 \mathrm{~mm}$ the intensity distribution in the HAADF image mainly consists of the average atomic number $Z^{1.65}$ of the probed volume ( $Z$-contrast) and the thickness of the specimen [28]. The GB-phase shows a double contrast with a dark interface to the adjacent grains and a bright center in the HAADF image in Figure 4(b). The HAADF intensity 


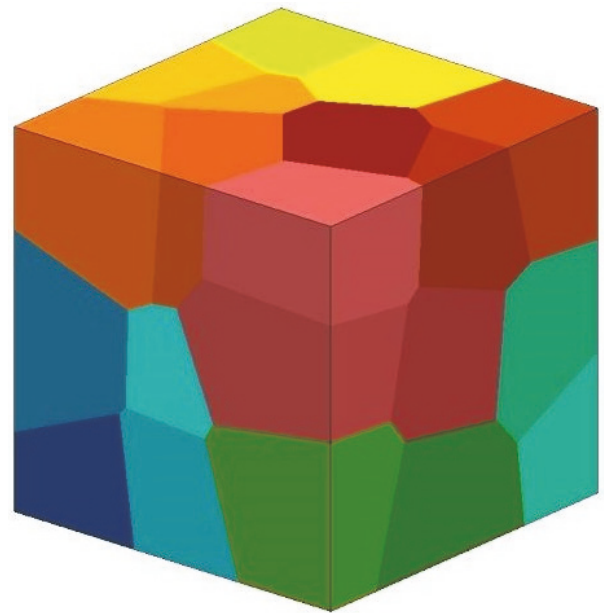

(a)

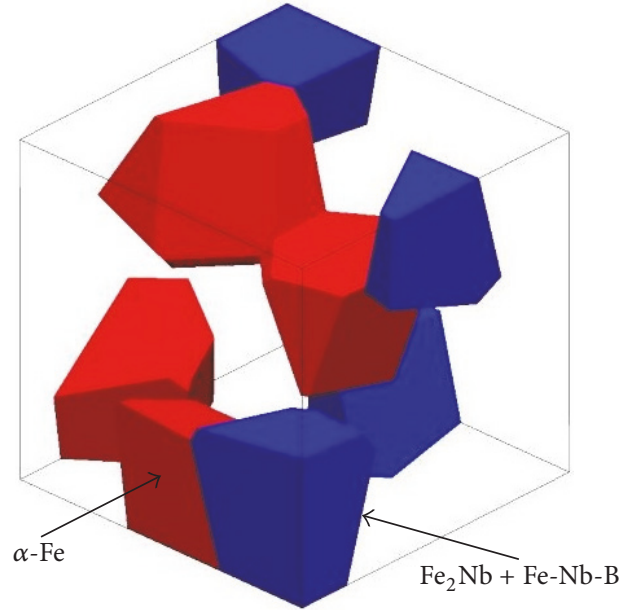

(b)

Figure 2: (a) Micromagnetic finite element model structure with 29 directly coupled Voronoi grains and (b) four $\alpha$-Fe grains (red) and four $\mathrm{Fe}-\mathrm{Nb}-\mathrm{B}$ grains (blue).

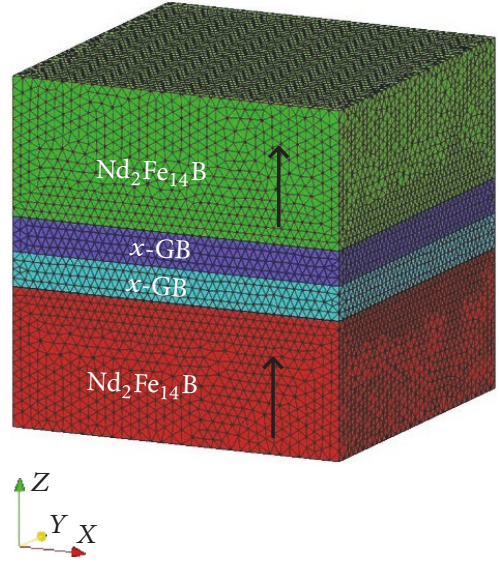

(a)

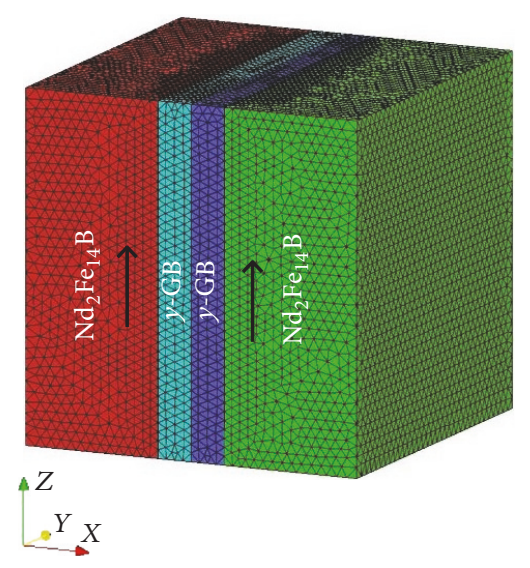

(b)

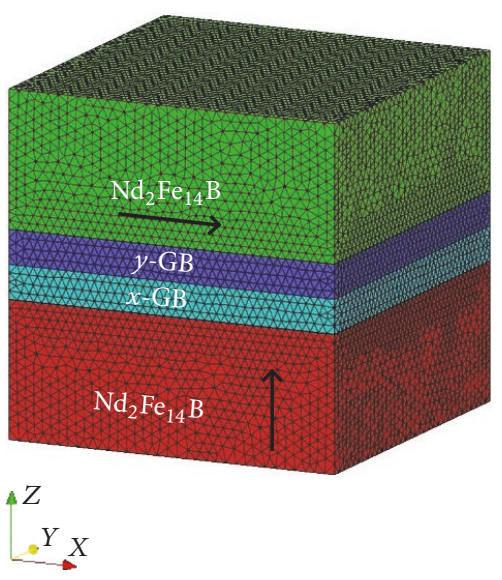

(c)

Figure 3: Micromagnetic finite element model structure with two $\mathrm{Nd}_{2} \mathrm{Fe}_{14} \mathrm{~B}$ grains separated by a 2-phase GB, (a) "aligned" magnet with $\mathrm{GB} \perp$ to [001]: $x$-GB, (b) "aligned" magnet with GB $\|$ to [001]: $y$-GB, and (c) "random" magnet with mixed GB-phase: $x$-GB $\perp$ to [001] and $y$-GB $\|$ to [001].

profile along the EELS-1 line scan and $Z^{1.65}$ dependence ( $Z$-contrast) are shown in the insert in Figure 4(b). The $Z$-contrast was calculated from the atomic percentage of the elements measured with EELS (Figure 7(a)). The dark interface between the grains and the GB is enriched in "Fe $+\mathrm{Co}$ " and contains less "Pr $+\mathrm{Nd}$," leading to a lower average atomic number. The $c$-axis of elongated grains was always found to be perpendicular to the longer edge of the grains.

The hard magnetic Nd-Fe-B grains are separated by a $3 \mathrm{~nm}-6 \mathrm{~nm}$ thick rare earth- (RE-) rich GB-phase and near GB junctions by the cubic $c-(\mathrm{Pr}, \mathrm{Nd})_{2} \mathrm{O}_{3}$ phase, which also has previously been reported in literature [2, 7, 11, 29-32]. The weakly paramagnetic $c-(\mathrm{Pr}, \mathrm{Nd})_{2} \mathrm{O}_{3}$ phase has only a negligible influence on the magnetization reversal compared to the soft ferromagnetic GB-phases. Dopants like Al, Ga, and $\mathrm{Cu}$ influence the liquid phase during sintering [3]. Ga-atoms were dissolved in the hard magnetic grains and GBs partially replacing the Fe-atoms during rapid quenching, since their amount is too low to form separate phases. The amorphous oxygen containing RE-rich GB-phase, shown in the HRTEM image in Figure 5, has an approximate composition of $(\mathrm{Pr}, \mathrm{Nd})_{41}(\mathrm{Fe}, \mathrm{Co})_{49} \mathrm{O}_{6} \mathrm{~F}_{4}$. The $\mathrm{RE} / \mathrm{Fe}$ ratio is in agreement with the composition of $\mathrm{Nd}_{48} \mathrm{Fe}_{48} \mathrm{Cu}_{4}$ reported by Sasaki et al. [33]. A combined STEM and three-dimensional atom probe tomography (3D-AP) study of sintered Nd-Fe-B magnets reported a chemical composition of the $\mathrm{Nd}$ enriched amorphous GB-phase of $\mathrm{Nd}_{30} \mathrm{Fe}_{45} \mathrm{Cu}_{24.1} \mathrm{~B}_{0.9}$ [34]. SepehriAmin et al. [35] produced a ferromagnetic $\mathrm{Nd}_{30} \mathrm{Fe}_{66} \mathrm{~B}_{3} \mathrm{Cu}_{1}$ thin film, whose chemical composition was derived from a laser assisted 3D-AP investigation of GB-phases of sintered $\mathrm{Nd}-\mathrm{Fe}-\mathrm{B}$ magnets. Woodcock et al. [36] reported of an amorphous oxide containing RE-rich GB-phase in a hot deformed 


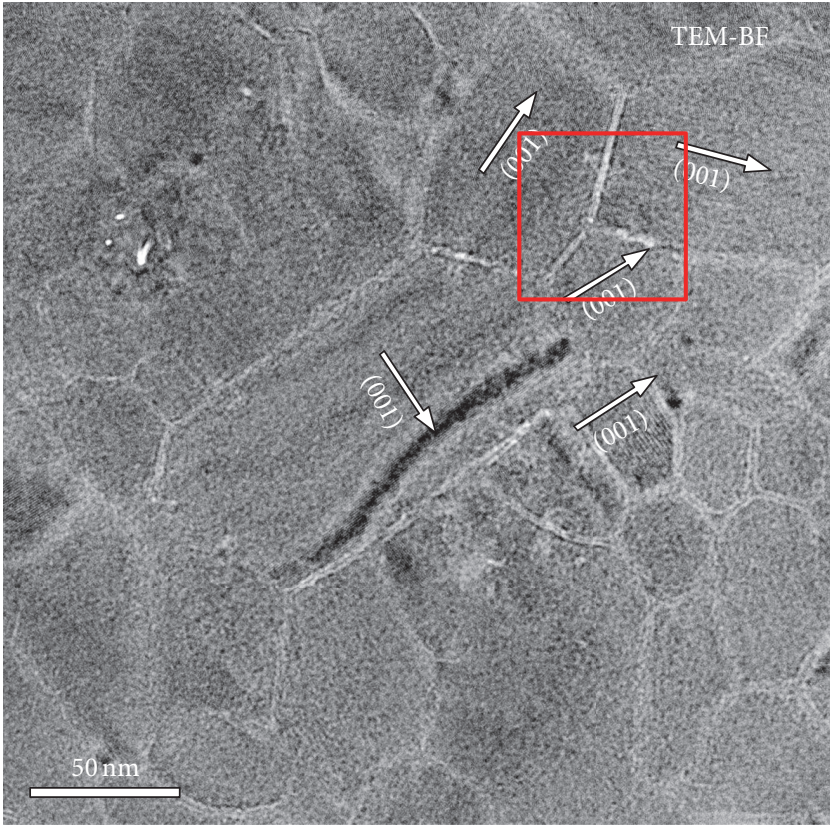

(a)

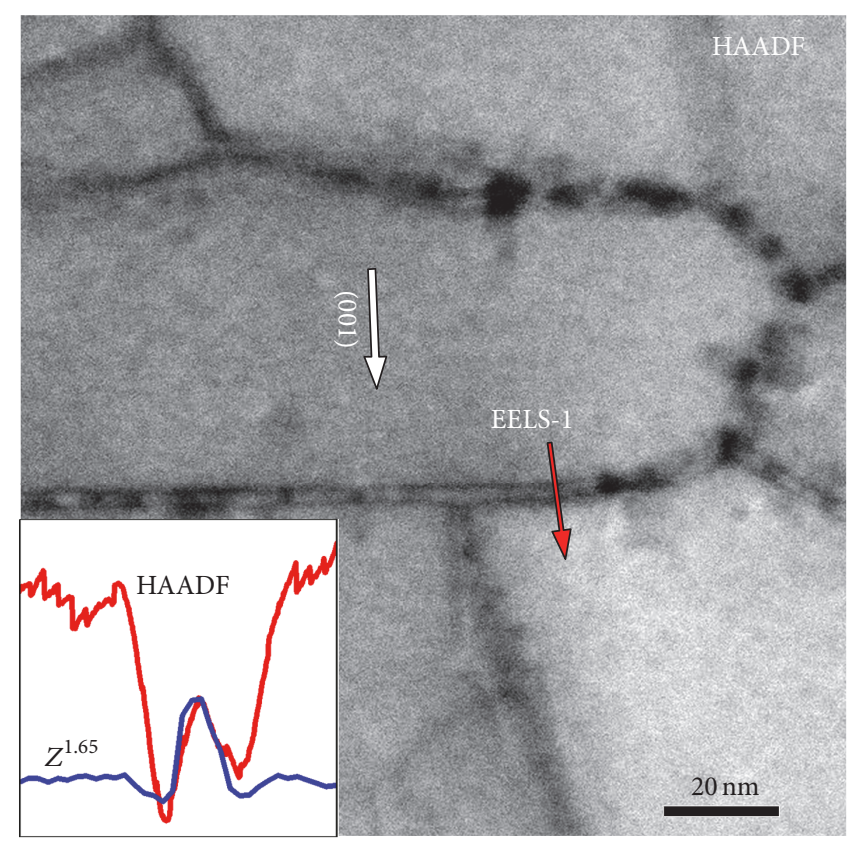

(b)

FIGURE 4: (a) TEM-BF image showing several misaligned grains with the marked [001] directions and the framed section of the HRTEM image of Figure 6. (b) HAADF image $(\mathrm{cl}=30 \mathrm{~mm}$ ) with the EELS-1 line scan (Figure 7) across GB with a double contrast. Insert in (b) correlates the double contrast of the GB (HAADF signal (red)) and the average $Z^{1.65}$ (blue) along the EELS-1 line scan.

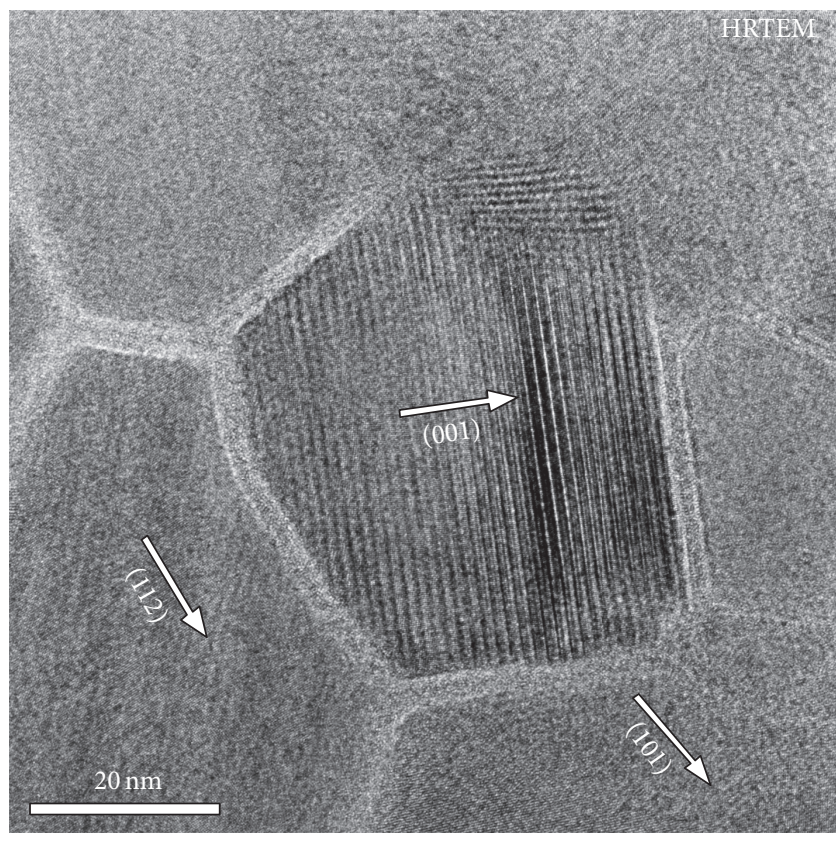

FIGURE 5: HRTEM image of randomly orientated grains separated by amorphous GBs. The (001), (112), and (101) lattice fringes of three grains are visible.

Nd-Fe-Co-B-Ga magnet. The (001), (112), and (101) lattice fringes of the hard magnetic $\mathrm{Nd}_{2} \mathrm{Fe}_{14} \mathrm{~B}$ grains are visible in Figure 5.

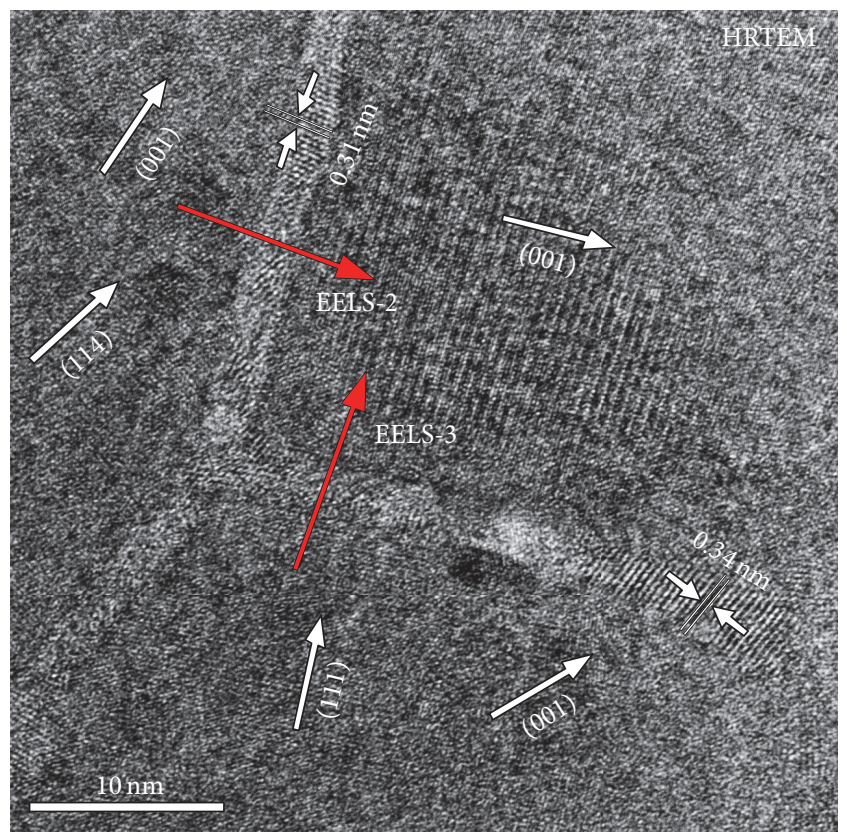

FIGURE 6: HRTEM image of three grains separated by crystalline GBs showing the (001) lattice fringes of the top right grain, (114) of the left grain, and (111) of the bottom grain are visible; the positions of the EELS line scans 2 and 3 of Figure 7 are shown.

Crystalline GBs with a lattice plane spacing of $0.31 \mathrm{~nm}$ and $0.34 \mathrm{~nm}$ are shown in the HRTEM image in Figure 6. The (001), (114), and (111) lattice fringes of the hard 


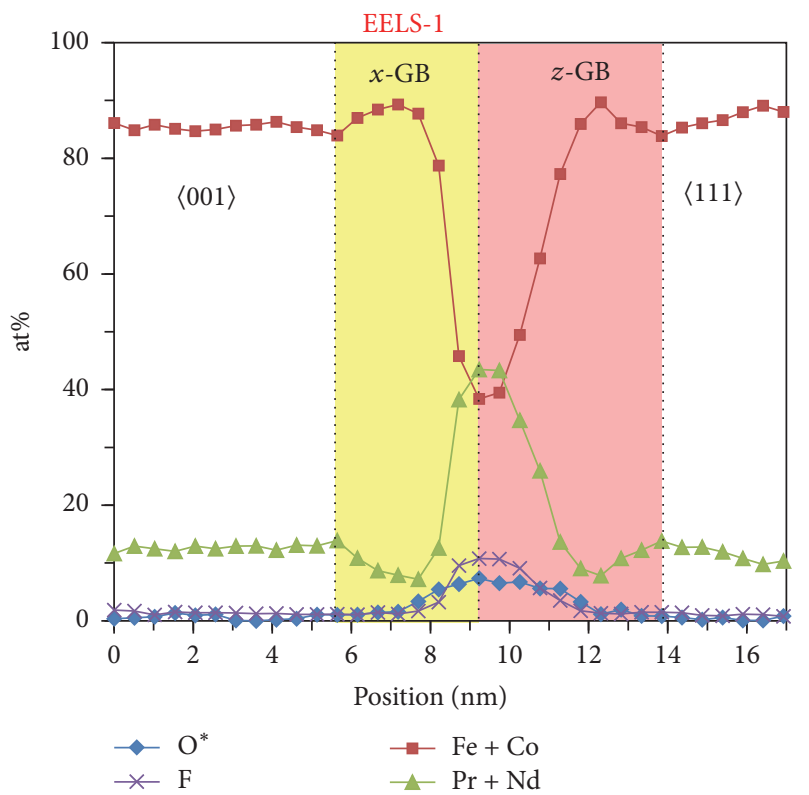

(a)

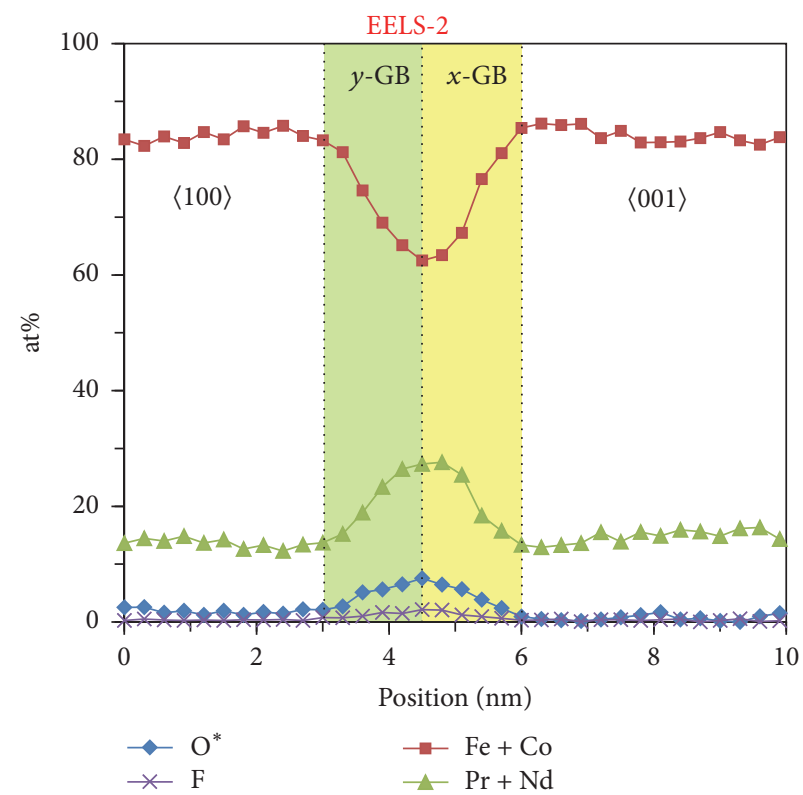

(b)

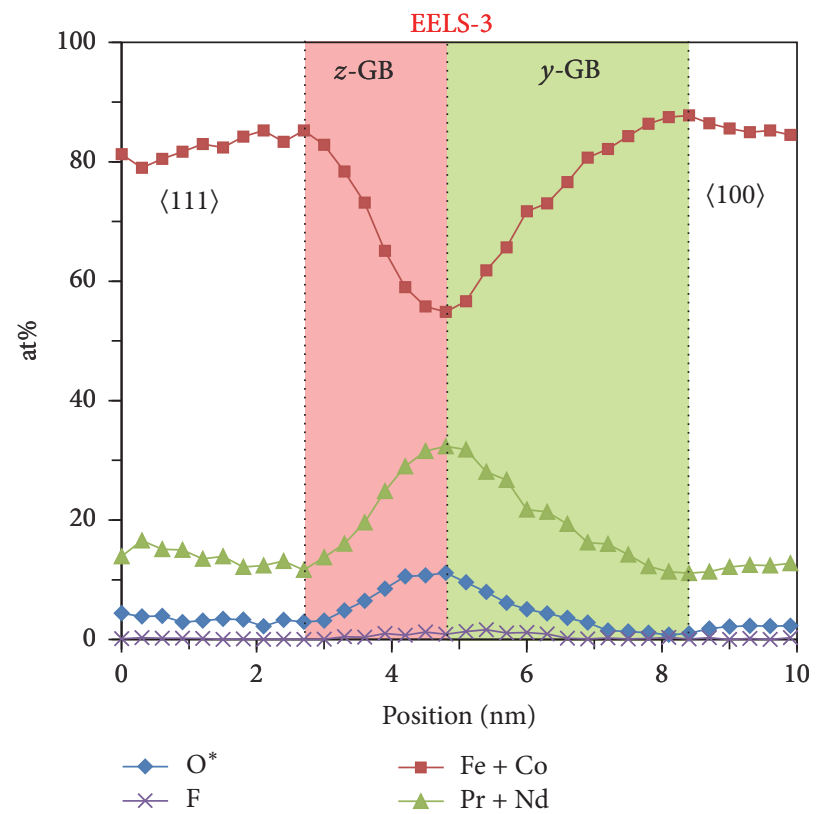

(c)

FIGURE 7: EELS line scans across GBs showing the asymmetric change of composition depending on the relative orientation of the GBs with respect to the neighbouring hard magnetic grains. (a) EELS-1 transition between $\langle 001\rangle$ and $\langle 111\rangle$ orientation $(x-z-G B)$. (b) EELS-2 line scan across the asymmetric $y-x$-GB (from $\langle 100\rangle$ to $\langle 001\rangle$ ) and (c) EELS-3 line scan across $z-y$-GB (from $\langle 111\rangle$ to $\langle 100\rangle$ ). The change in the chemical composition from the 2-14-1 grain into the GB is steeper in the $x$-GB compared to $z$-GB in (a); in the $x$-GB compared to the $y$-GB in (b); and in the $z$-GB compared with the $y$-GB in (c).

magnetic grains are visible. Sasaki et al. [37] reported about a crystalline GB-phase with a $\mathrm{RE}$ content of 60 at\% in $\mathrm{Nd}_{13.5} \mathrm{Pr}_{0.2} \mathrm{Dy}_{0.2} \mathrm{~Tb}_{0.2} \mathrm{Fe}_{76.0} \mathrm{Co}_{1.8} \mathrm{~B}_{6.6} \mathrm{Cu}_{0.1} \mathrm{Al}_{0.5} \mathrm{Ni}_{0.4} \mathrm{O}_{0.5}$ sintered magnet with a high energy product investigated with STEM methods. Another 3D-AP study [33] of a sintered Nd-
Fe-B magnet reported about a crystalline GB with Nd-content of 55 at\%. A crystalline $5 \mathrm{~nm}-10 \mathrm{~nm}$ thick $\mathrm{Cu}$ enriched cubic c- $\mathrm{Nd}_{2} \mathrm{O}_{3}$ GB-phase in $\mathrm{Nd}_{12.0} \mathrm{Dy}_{2.7} \mathrm{Fe}_{76.3} \mathrm{Cu}_{0.4} \mathrm{~B}_{6.0} \mathrm{M}_{2.6}(\mathrm{M}=$ $\mathrm{Al}$, Co, and $\mathrm{Nb}$ ) sintered $\mathrm{Nd}-\mathrm{Fe}-\mathrm{B}$ magnet was reported by Kim et al. [38]. A crystalline $\mathrm{Nd}$ enriched 
$\mathrm{Nd}_{16.4} \mathrm{Fe}_{71.8} \mathrm{Co}_{7.8} \mathrm{~B}_{3.5} \mathrm{Ga}_{0.5} \mathrm{~GB}$-phase in $\mathrm{Nd}-\mathrm{Fe}-\mathrm{B}$ magnets subjected to a hydrogen-disproportion-desorption-recombination process was reported in 3D-AP study [39].

In a previous study we have shown [11] that in an aligned sintered magnet the GBs perpendicular $(x-\mathrm{GB})$ to the alignment direction of the magnet have a higher RE content (up to 60 at $\%$ ) than the GBs parallel $(y-\mathrm{GB})$ to the alignment direction (RE content below 30 at $\%$ ). GBs with intermediate misorientation to the alignment direction ( $z$-GB) show a chemical composition corresponding to an average of $x$ - and $y$-GB. In sintered anisotropic magnets pure $x$ - and $y$-GBs are common, but in melt-spun isotropic magnet materials the GB is a mix of $x$ - and $y$-GB in general, due to the strong misalignment of the neighbouring grains. The EELS-1 line scan starts from a 2-14-1 grain into a $x$-GB, resulting in a strong gradient of the chemical composition, and continues from the $z$-GB into a grain with approximately $45^{\circ}$ misorientation of the $c$-axis with respect to the surface normal of the GB (Figures 4(b) and 7(a)). This correlates with a gradual change of the chemical composition. The EELS-2 line scan starts in a grain whose $c$-axis is orientated perpendicular to the surface normal of the GB resulting in a slow change in chemical composition (Figures 6 and 7(b)). Since the $c$-axis of the second grain is orientated parallel to the surface normal of the GB the change in chemical composition is faster. The faster change in the chemical composition from a $z$-GB with respect to the $y$-GB is shown in the EELS-3 line scan (Figures 6 and $7(\mathrm{c})$ ).

The average "Fe + Co" concentration of the GB-phase in the investigated MQU-F ribbon is 55 at $\%$, if only "Fe $+\mathrm{Co}$ " and "Pr $+\mathrm{Nd}$ " elements are considered. According to the magnetic phase diagram of $\mathrm{Nd}_{100-x} \mathrm{Fe}_{x}$ which was recently published by Sakuma et al. [40] we assumed for the GB-phase a magnetic saturation polarization $J_{s}$ of $0.43 \mathrm{~T}$ and calculated an exchange stiffness constant $A$ of $1.0 \mathrm{pJ} / \mathrm{T}$. The relation $A \propto$ $\varepsilon \cdot J_{s}^{2}$ between $J_{s}$ and the exchange constant $A$ was used, as suggested by Kronmüller and Fähnle [41].

Using the Voronoi model structure of isotropically orientated $\mathrm{Nd}_{2} \mathrm{Fe}_{14} \mathrm{~B}$ grains (Figure 1) with an average grain size of $50 \mathrm{~nm}$ and a GB-phase with a thickness of $4 \mathrm{~nm}-6 \mathrm{~nm}$ (Figures 5 and 6) we calculated the demagnetization curves obtained from the numerical finite element micromagnetic simulations depending on the coupling between the grains and the degree of misorientation of the grains. Figure 8 shows a high accordance of the coercive field $H_{c J}$ between the measured value and the randomly misoriented grains. It should be noted that for the simulated demagnetization curve (sm-GB_60 $0^{\circ}$ the remanence $J_{r}$ gets underestimated in the simulation with a perfectly isotropic distribution of the $c$-axes $\left(\vartheta_{0} \approx 60^{\circ}\right)$. In addition Figure 8 shows that the simulations for directly coupled $\mathrm{Nd}_{2} \mathrm{Fe}_{14} \mathrm{~B}$ grains (no-GB-phase) underestimate the coercive field by $1.5 \mathrm{~T}\left(\vartheta_{0} \approx 60^{\circ}\right)$. The simulation with a smaller degree of misalignment of the hard magnetic grains $\left(\vartheta_{0} \approx 45^{\circ}\right)$ reveals the significant increase of $H_{c J}$ and $J_{r}$ with respect to the perfectly isotropically oriented case $\left(\vartheta_{0} \approx 60^{\circ}\right)$. This is in agreement with the Stoner-Wohlfarth model of noninteracting single-domain particles [26], where $H_{c J}$ is increasing by $\approx 5 \%$ of the anisotropy field $H_{A}$, which

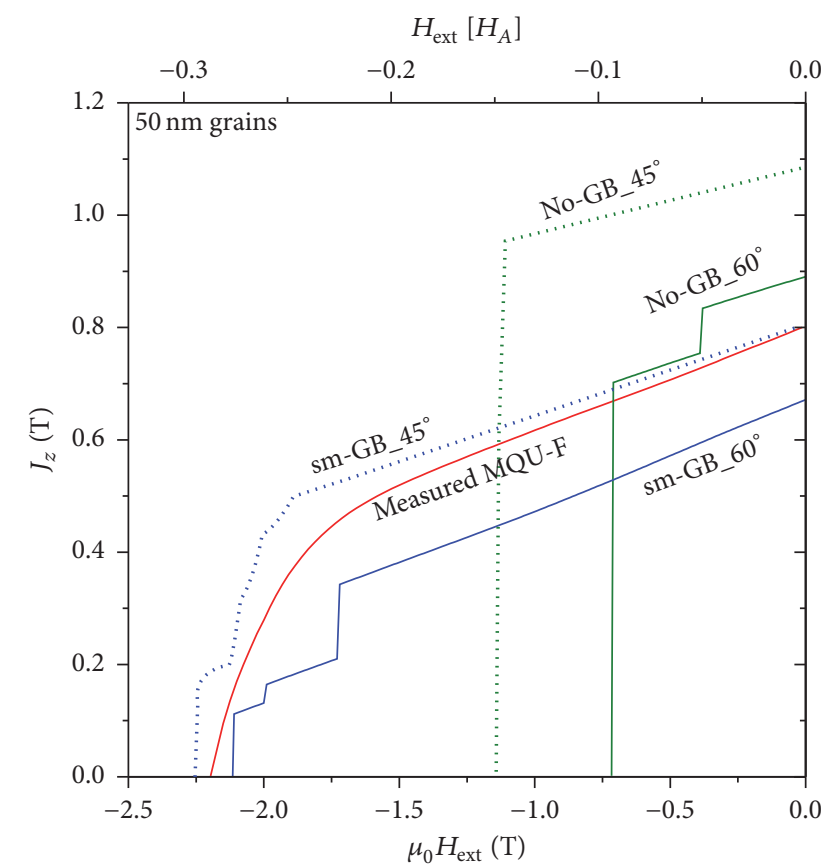

FIGURE 8: Comparison of the measured demagnetization curve of the MQU-F melt-spun ribbon with calculated curves for directly coupled $\mathrm{Nd}_{2} \mathrm{Fe}_{14} \mathrm{~B}$ grains (no-GB) and grains separated by a weakly soft magnetic GB-phase (sm-GB) with $J_{s}=0.43 \mathrm{~T}$ and $A=1.0 \mathrm{pJ} / \mathrm{T}$ for an average grain misorientation of $45^{\circ}$ and $60^{\circ}$. The average grain size is $50 \mathrm{~nm}$ and the average GB thickness is $5 \mathrm{~nm}$.

corresponds to $\approx 0.4 \mathrm{~T}$ in $\mathrm{Nd}_{2} \mathrm{Fe}_{14} \mathrm{~B}$, if $\vartheta_{0}$ is reduced from $60^{\circ}$ to $45^{\circ}$. The reduction of $H_{c J}$ with rising value of $\vartheta_{0}$ is attenuated in the simulations with a ferromagnetic GB-phase. The higher $J_{r}$ value of the simulation with $\vartheta_{0} \approx 45^{\circ}$ with respect to the simulation with $\vartheta_{0} \approx 60^{\circ}$ is explained by the higher value of the component of the polarization parallel to the applied field direction ( $z$-direction).

4.1.2. Isotropic Fine Grained Melt-Spun Ribbon (MQP-B+). The small grained microstructure of the sample MQP-B+ is shown in the TEM-BF image of Figure 9(a). The isotropic orientation of the $c$-axes of the $\mathrm{Nd}-\mathrm{Fe}-\mathrm{B}$ grains with a grain size ranging from $15 \mathrm{~nm}$ to $50 \mathrm{~nm}$ is displayed in the medium angle annular dark field image (MAADF) of Figure 9(b), which is generated at a higher camera length $(\mathrm{cl}=970 \mathrm{~mm})$ compared to the HAADF image. The MAADF contrast generation is similar to the one of a TEM-BF image. The insert in Figure 9(a) shows EELS line scan across a $3 \mathrm{~nm}$ thick " $\mathrm{Pr}+$ Nd" enriched GB-phase.

Under the assumption that all boron is bound in the $\mathrm{Nd}_{2}(\mathrm{Fe}, \mathrm{Co})_{14} \mathrm{~B}$ phase the chemical composition of the intergranular GB-phases has been calculated from the nominal composition $\mathrm{Nd}_{12.4}(\mathrm{Fe}, \mathrm{Co})_{82.5} \mathrm{~B}_{5.2}$ to be $\mathrm{Nd}_{17}(\mathrm{Fe}, \mathrm{Co})_{83}$. This corresponds to 12 at $\%$ of the total composition. With the approximation of $30 \mathrm{~nm}$ large rhombic dodecahedron shaped grains separated by a $2 \mathrm{~nm}-3 \mathrm{~nm}$ thick GB-phase the volume fraction of the GB-phase is $21 \%$. The chemical composition of the GB measured by EELS is $\mathrm{Nd}_{20}(\mathrm{Fe}, \mathrm{Co})_{77} \mathrm{O}_{3}$. These results 


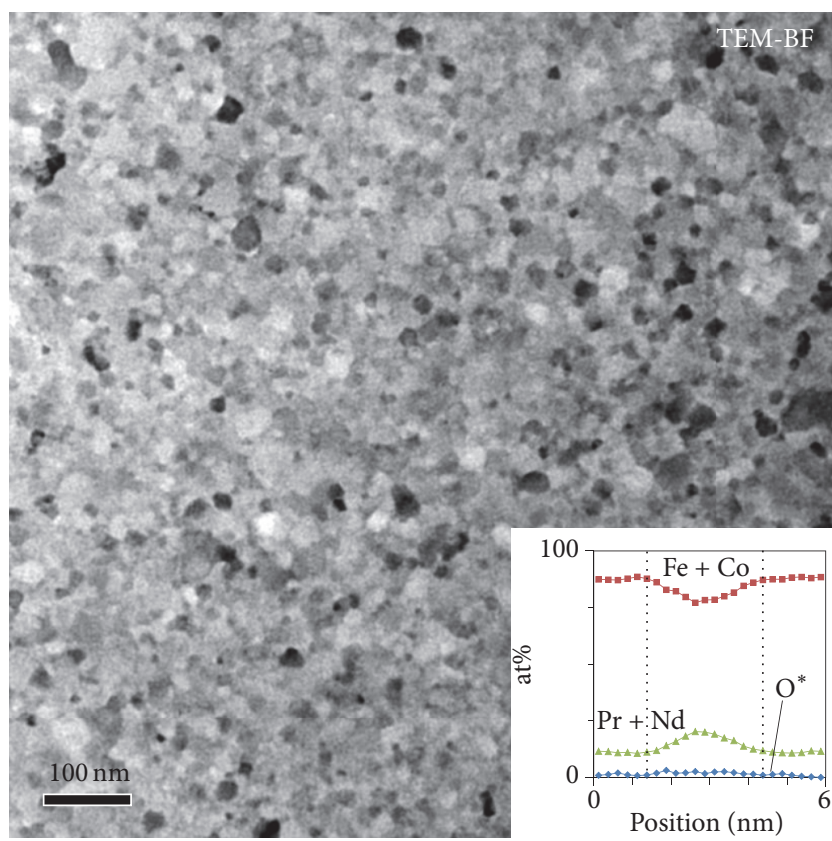

(a)

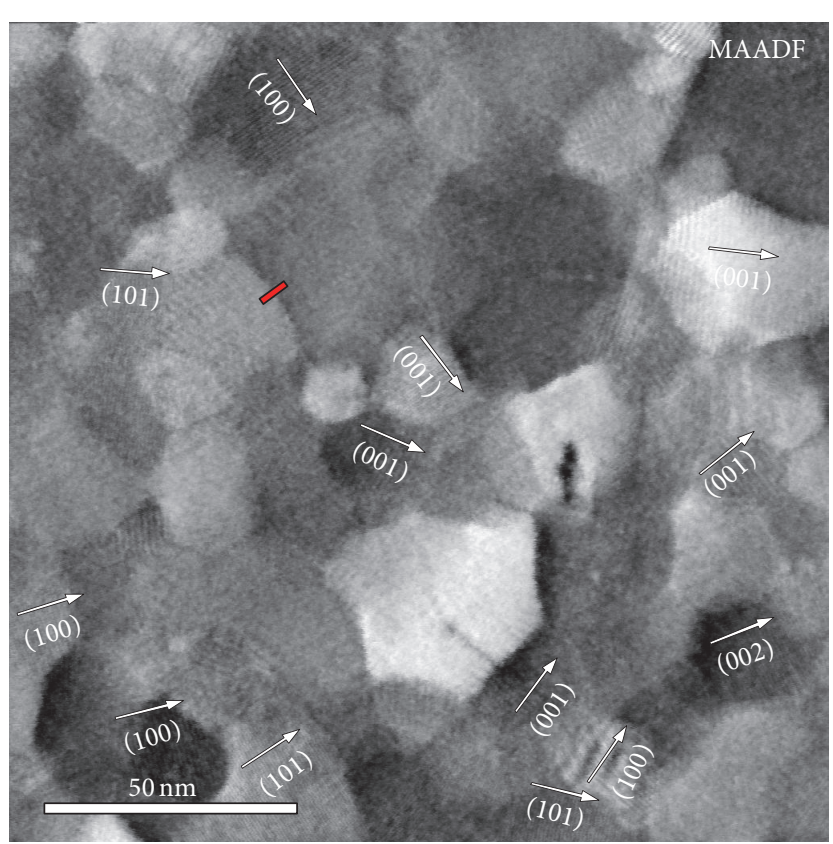

(b)

Figure 9: (a) TEM bright field (BF) image of small grained MQP-B+ magnet, [(a) insert] EELS line scan across a $3 \mathrm{~nm}$ thick "Pr $+\mathrm{Nd}$ " enriched GB-phase, and (b) MAADF ( $\mathrm{cl}=970 \mathrm{~mm})$ image with an isotropic distribution of the (001) lattice plane normal of the 2-14-1 grains and the EELS scan of Figure 9(a) (red line).

are in good agreement with experiments with an Auger Microprobe spectrometer [42].

The micromagnetic simulations were carried out with the Voronoi model structure with isotropically orientated grains (Figure 1) with an average grain size of $35 \mathrm{~nm}$ and a soft magnetic GB-phase with a thickness of $2 \mathrm{~nm}-4 \mathrm{~nm}$ and average values for $J_{s}=1.1 \mathrm{~T}$ and $A=6.54 \mathrm{pJ} / \mathrm{m}$, which is similar as described for the MQU-F sample. The simulated coercive field value is in good agreement with the measured value (Figure 10). Due to the high $J_{s}$ value of the GB the coercive field value (sm-GB) is only slightly increased with respect to $H_{c J}$ of the simulation from directly coupled $\mathrm{Nd}_{2} \mathrm{Fe}_{14} \mathrm{~B}$ grains (no-GB).

4.1.3. Isotropic Large Grained Nanocomposite with $\alpha$-Fe and $\mathrm{Nb}$-Containing Granular Phases. The large grained microstructure of the exchange coupled nanocomposite with isotropically orientated $\mathrm{Nd}-\mathrm{Fe}-\mathrm{B}$ grains and a grain size ranging from $30 \mathrm{~nm}$ to $150 \mathrm{~nm}$ is shown in the TEM-BF image of Figure 11(a). The insert in Figure 11(a) is EELS line scan across a GB of two $\mathrm{Nd}_{2} \mathrm{Fe}_{14} \mathrm{~B}$ grains with no detected intergranular GB-phase. Besides the hard magnetic 2-14-1 phase the soft ferromagnetic $\alpha$-Fe and the weakly antiferromagnetic $\mathrm{Fe}_{2} \mathrm{Nb}$ phase $\left(T<T_{N} \approx 270 \mathrm{~K}\right)[43]$ are shown in the HRTEM image in Figure 11(b).

A large area EDX mapping in the HAADF image in Figure 12(b)-12(e) was used to determine the areal fraction of the identified granular phases (Figure 12(a)). Besides the

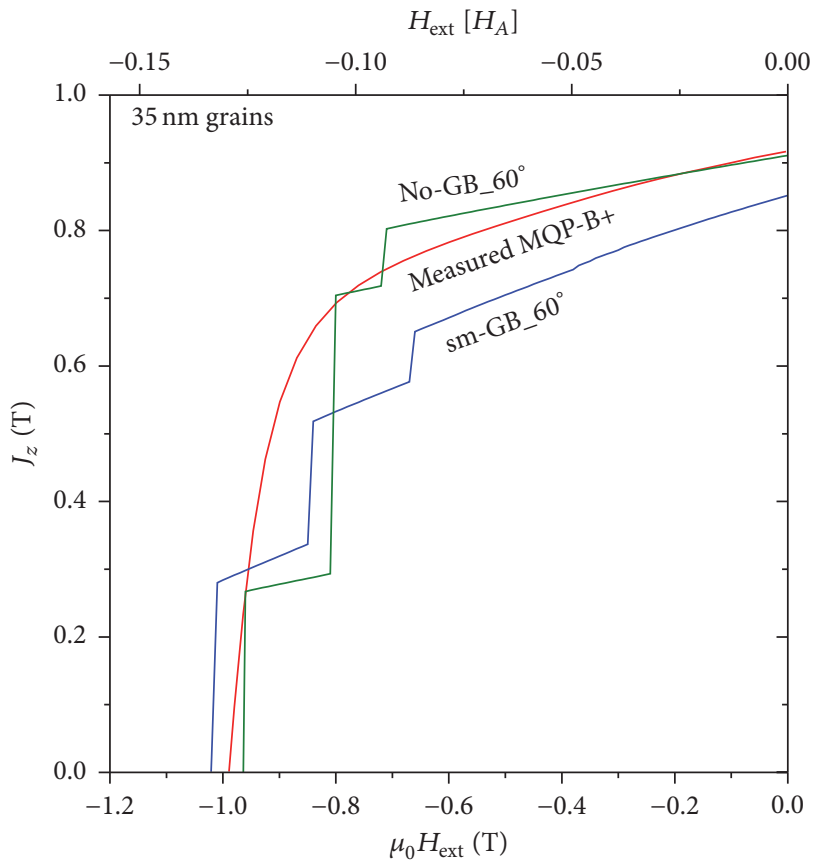

FIGURE 10: Comparison of the measured demagnetization curve of the MQP-B+ melt-spun ribbon with calculated curves for directly coupled $\mathrm{Nd}_{2} \mathrm{Fe}_{14} \mathrm{~B}$ grains (no-GB) and grains separated by a weakly soft magnetic GB-phase (sm-GB) with $J_{s}=1.1 \mathrm{~T}$ and $A=6.54 \mathrm{pJ} / \mathrm{T}$ for an average grain misorientation of $60^{\circ}$. The average grain size is $35 \mathrm{~nm}$ and the average GB thickness is $3 \mathrm{~nm}$. 


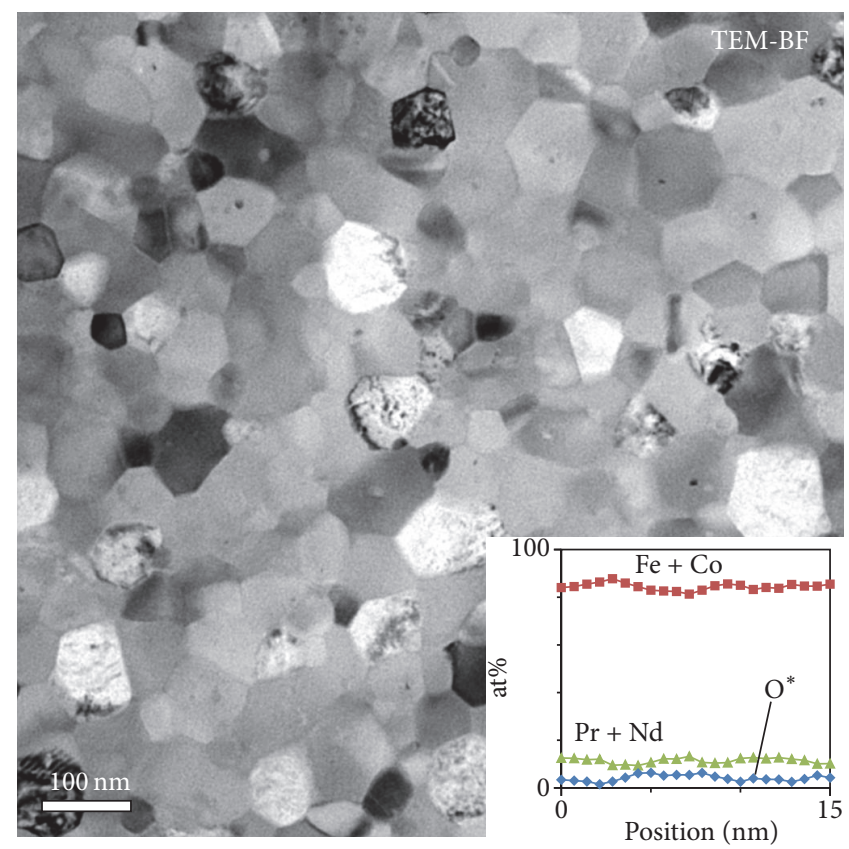

(a)

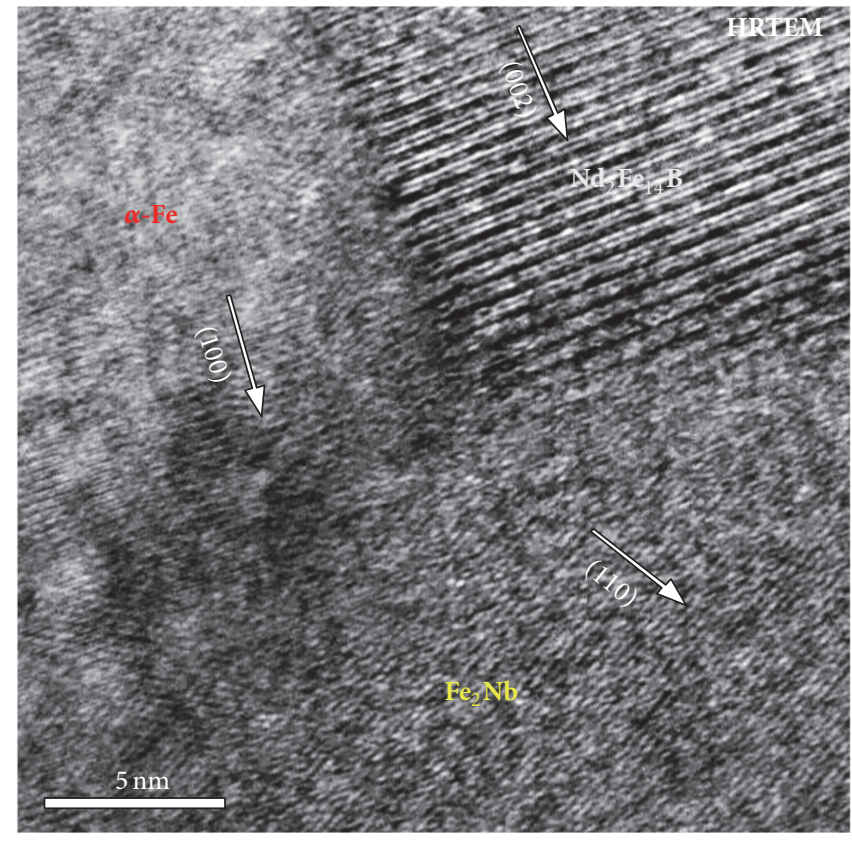

(b)

FIGURE 11: (a) TEM-BF image of the multiphase large grained nanocomposite. ((a) insert) No change in chemical composition in the EELS line scan across a GB. No separate GB-phase is detected. (b) HRTEM image of three granular phases.

$\alpha$-Fe phase another soft magnetic $\mathrm{Nb}_{6} \mathrm{Fe}_{76} \mathrm{~B}_{18}\left(J_{s}=1.41 \mathrm{~T}\right.$, $H_{c J}=2.8 \mathrm{mT}$ ) phase which was formed by rapid quenching [44] was identified. Table 1 summarizes the lattice parameter, space groups, and prototypes of the analyzed phases which were used to identify the phases in the HRTEM images. The bright areas in the Fe-K map (Figure 12(c)) correspond to the $\alpha$-Fe phase. The $\mathrm{Fe}_{2} \mathrm{Nb}$ phase is located at the high intensities of the $\mathrm{Nb}-\mathrm{K}$ map (Figure $12(\mathrm{~d})$ ) and the $\mathrm{Nb}_{6} \mathrm{Fe}_{76} \mathrm{~B}_{18}$ phase at the more dull yellow regions. The location of the 2-14-1 phase is clearly visible in the bright areas in the $\mathrm{Nd}-\mathrm{L}$ map (Figure 12(e)).

A Voronoi model structure with 29 directly coupled grains (Figure 2) with an average size of $60 \mathrm{~nm}$ was used to simulate the hysteretic properties. Corresponding to the analyzed volume distribution of the phases we assumed 21 $(72 \%) \mathrm{Nd}_{2} \mathrm{Fe}_{14} \mathrm{~B}$ grains, 4 (14\%) $\alpha$-Fe grains, and $4(14 \%)$ $\mathrm{Nb}_{6} \mathrm{Fe}_{76} \mathrm{~B}_{18}$ grains. The magnetic properties of the phases are summarized in Table 2 . All $K_{1}$ values were set to zero except in the hard magnetic $\mathrm{Nd}_{2} \mathrm{Fe}_{14} \mathrm{~B}$ phase.

The measured demagnetization curve and the simulated curves of directly coupled grains with an average grain misorientation of $45^{\circ}$ and $60^{\circ}$ are shown in Figure 13. For the realistic phase distribution the calculated coercive field is slightly underestimated in the simulation compared to the measured value. One reason for this discrepancy is relatively small sample area where the areal distribution was acquired, with respect to the whole ribbon volume. A higher quality of the random distribution of the granular phases would be achieved in a model with a larger number of grains. The model with 29 directly coupled $\mathrm{Nd}_{2} \mathrm{Fe}_{14} \mathrm{~B}$ grains overestimates both $H_{c J}$ and $J_{r}$ significantly. The strong decrease of $H_{c J}$ in the model structure with the realistic assumption of soft magnetic grains, compared to the case of only hard magnetic $\mathrm{Nd}_{2} \mathrm{Fe}_{14} \mathrm{~B}$ grains, was also reported in a detailed micromagnetic study of $\mathrm{Nd}-\mathrm{Fe}-\mathrm{B}$ magnet with soft magnetic granular phases [45].

4.2. Micromagnetic Simulations of the Switching Field of Randomly Orientated Grains. The orientation relation of grain boundaries of adjacent grains and their composition close to their grain surfaces with respect to the alignment direction of the magnet and external field direction influence the resulting magnetic switching field and coercive field, respectively. Using the two-grain (2-G) model structure of Figure 3 we compare in Figure 14 three different configurations which possibly occur in anisotropically and isotropically oriented magnets. The first and second case in Figure 14 show a pure $x$ $\mathrm{GB}$ and pure $y$-GB, commonly found in anisotropic aligned sintered Nd-Fe-B magnets. The external field is parallel to [001] direction in both cases. The third case shows $x$-GB facing the lower grain and $y$-GB facing the upper grain and $H_{\text {ext }}$ is parallel to [111], typically found in isotropically oriented melt-spun Nd-Fe-B magnets.

$J_{s}$ values for $x$ - and $y$-GB were calculated from the chemical composition obtained from TEM/EELS measurements of GBs in anisotropic sintered Nd-Fe-B magnets [11]. The

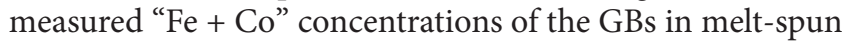
magnets (Figures 7 and 9(a)) and the corresponding $J_{s}$ and $A$ values are summarized in Table 3.

The micromagnetic simulations show that the switching field $H_{\text {sw }}$ depends on both, the GB thickness and $J_{s}$ value of the GB layer (Figure 15(a)). For small $J_{s}$ value of the $x$-GB 


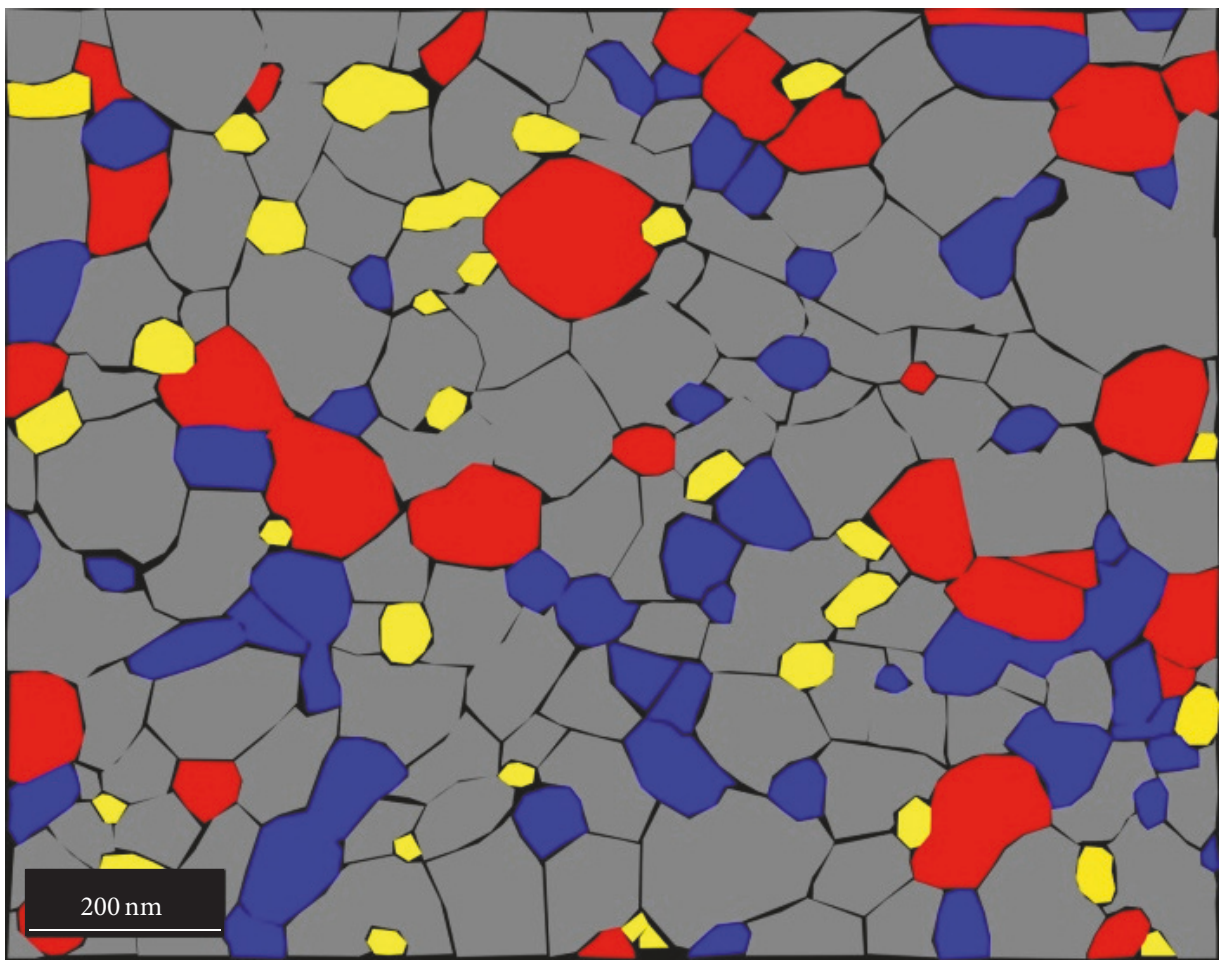

(a)

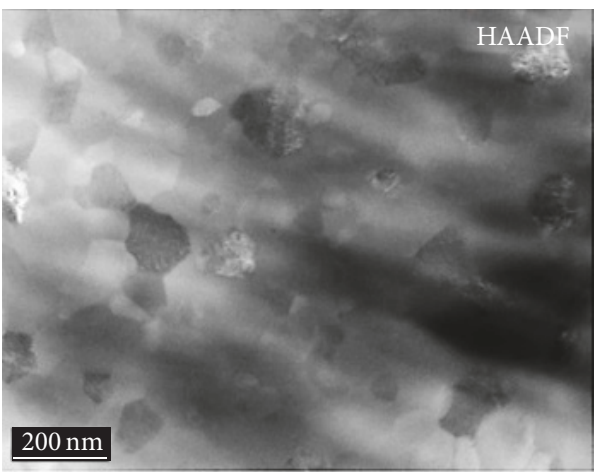

(b)

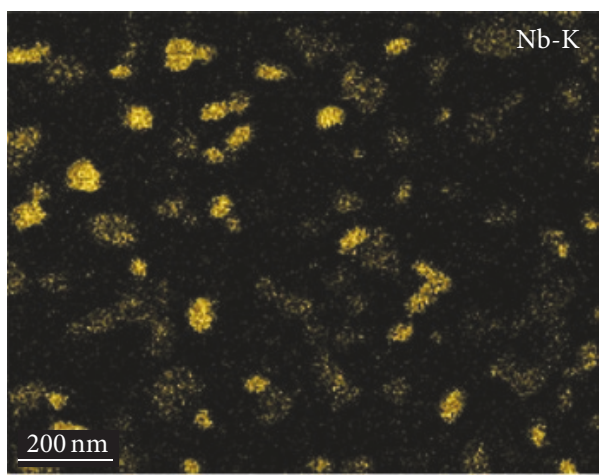

(d)

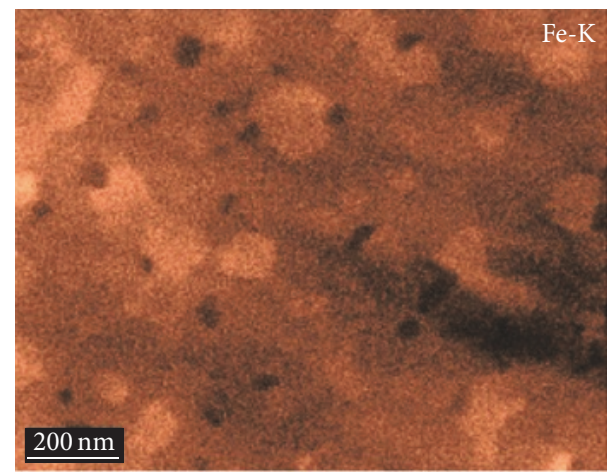

(c)

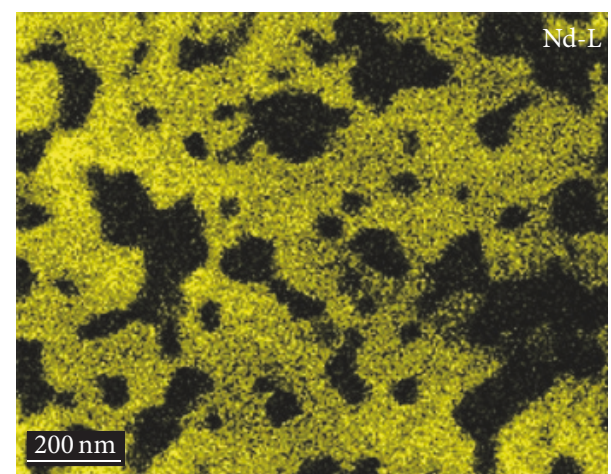

(e)

FIGURE 12: (a) Morphological map of the four identified granular phases obtained from TEM analysis. (b) HAADF image (cl = $200 \mathrm{~mm}$ ). (c-e) elemental (Fe, Nb, and Nd) EDX maps of sample area as shown in (b). $\mathrm{Nd}_{2} \mathrm{Fe}_{14} \mathrm{~B}$ (grey), $\alpha$ - $\mathrm{Fe}$ (red), $\mathrm{Nb}_{6} \mathrm{Fe}_{76} \mathrm{~B}_{18}$ (blue), and $\mathrm{Fe}_{2} \mathrm{Nb}$ (yellow). 
TABLE 1: Crystal structure and lattice parameters of identified phases in the large grained nanocomposite Nd-Fe-B melt-spun ribbon.

\begin{tabular}{|c|c|c|c|c|c|c|}
\hline \multirow{2}{*}{ Crystal structure } & \multicolumn{2}{|c|}{ Lattice parameter } & \multirow{2}{*}{\multicolumn{2}{|c|}{ Space group }} & \multirow{2}{*}{ Prototype } & \multirow{2}{*}{ Reference } \\
\hline & $a[\mathrm{~nm}]$ & $c[\mathrm{~nm}]$ & & & & \\
\hline $\mathrm{Nd}_{2} \mathrm{Fe}_{14} \mathrm{~B}$ & 0.8803 & 1.2196 & $\mathrm{P}_{2} / \mathrm{mnm}$ & 136 & $\mathrm{Nd}_{2} \mathrm{Fe}_{14} \mathrm{~B}$ & {$[46]$} \\
\hline$\alpha-\mathrm{Fe}$ & 0.2865 & - & $\operatorname{Im} \overline{3} m$ & 229 & W & {$[47]$} \\
\hline $\mathrm{Nb}_{6} \mathrm{Fe}_{76} \mathrm{~B}_{18}$ & 1.0819 & - & $F m \overline{3} m$ & 225 & $\mathrm{NaCl}$ & {$[44]$} \\
\hline $\mathrm{Fe}_{2} \mathrm{Nb}$ & 0.4835 & 0.7881 & $\mathrm{PG}_{3} / \mathrm{mmc}$ & 194 & $\mathrm{MgZn}_{2}$ & {$[48]$} \\
\hline
\end{tabular}

TABLE 2: Areal fraction and magnetic properties of the four identified granular phases used in the micromagnetic simulations.

\begin{tabular}{|c|c|c|c|c|c|}
\hline Phase & Areal fraction [\%] & $K_{1}\left[\mathrm{MJ} / \mathrm{m}^{3}\right]$ & $J_{s}[\mathrm{~T}]$ & $A[\mathrm{pJ} / \mathrm{m}]$ & Reference \\
\hline $\mathrm{Nd}_{2} \mathrm{Fe}_{14} \mathrm{~B}$ & 65 & 4.9 & 1.61 & 7.7 & {$[49,50]$} \\
\hline$\alpha-\mathrm{Fe}$ & 16 & 0 & 2.15 & 25.0 & {$[51]$} \\
\hline $\mathrm{Nb}_{6} \mathrm{Fe}_{76} \mathrm{~B}_{18}$ & 15 & 0 & 1.41 & 10.8 & {$[44]$} \\
\hline $\mathrm{Fe}_{2} \mathrm{Nb}$ & 4 & 0 & $0.00^{1}$ & $0.0^{1}$ & {$[43]$} \\
\hline
\end{tabular}

${ }^{1}$ The Néel temperature of the weakly antiferromagnetic $\mathrm{Fe}_{2} \mathrm{Nb}$ phase is $\approx 270 \mathrm{~K}$ and therefore we assumed nonmagnetic properties for the simulation at room temperature.

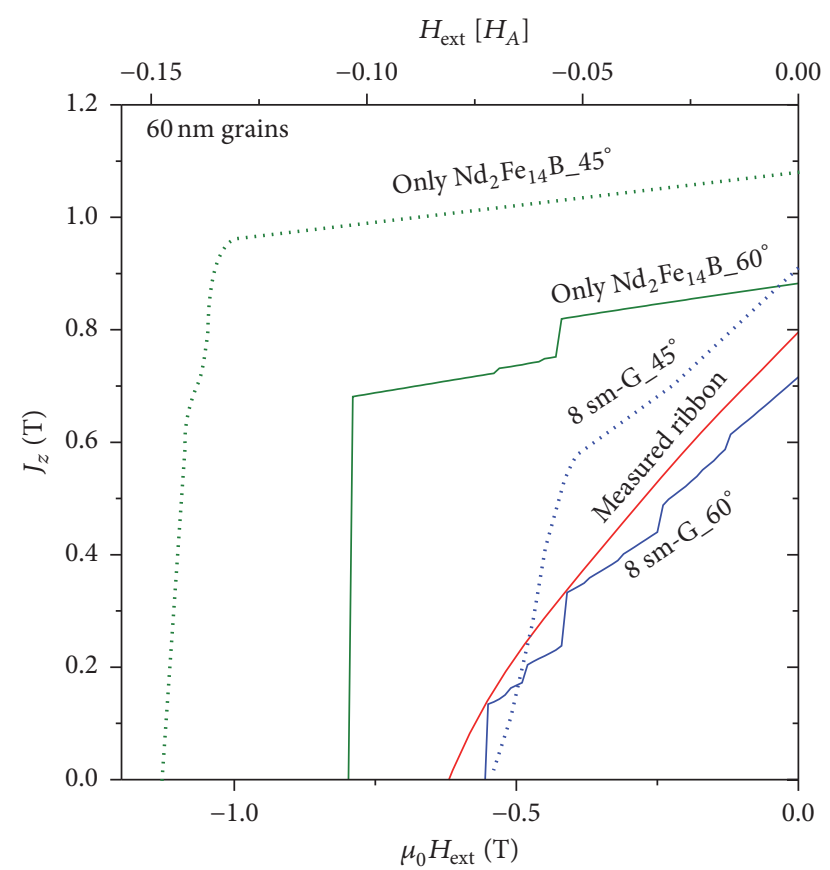

FIGURE 13: Comparison of the measured demagnetization curve of the $\mathrm{Nd}-\mathrm{Fe}-\mathrm{B}$ nanocomposite melt-spun ribbon with calculated curves for directly coupled only hard magnetic grains (only $\mathrm{Nd}_{2} \mathrm{Fe}_{14} \mathrm{~B}$ ) and for the model structure with 8 soft ferromagnetic grains and $21 \mathrm{Nd}_{2} \mathrm{Fe}_{14} \mathrm{~B}$ grains $(8 \mathrm{sm}-\mathrm{G}) .45^{\circ}$ and $60^{\circ}$ denote the average misorientation of the granular model structure. The average grain size is $60 \mathrm{~nm}$.

$(<0.2 \mathrm{~T}) H_{\mathrm{sw}}$ slightly increases with rising GB thickness $(x$ $\mathrm{GB})$. For high $J_{s}$ value of the $y-\mathrm{GB}(1.0 \mathrm{~T}) H_{\mathrm{sw}}$ is significantly lower with rising $\mathrm{GB}$ thickness $(y-\mathrm{GB})$. In both cases the external field is parallel to [001] direction. This behaviour
TABle 3: Measured Fe + Co content in GBs in sintered and meltspun Nd-Fe-B magnets and resulting magnetic properties.

\begin{tabular}{lccc}
\hline & Measured Fe + Co [at $\%]$ & $J_{s}[\mathrm{~T}]^{1}$ & $A[\mathrm{pJ} / \mathrm{m}]^{2}$ \\
\hline$x$-GB (sintered) & $40^{3}$ & 0.15 & 0.12 \\
$y$-GB (sintered) & $75^{3}$ & 1.00 & 5.41 \\
MQU-F & 55 & 0.43 & 1.00 \\
MQP-B+ & 79 & 1.10 & 6.54 \\
\hline
\end{tabular}

${ }^{1}$ After Sakuma et al. [40], ${ }^{2} A$ is proportional to $J_{s}^{2}[41] .{ }^{3}[11]$.

is typical for anisotropic magnets with perfectly aligned grains. In the isotropic case ( $x y$-GB), with $H_{\text {ext }} \|$ [111], the switching field value slightly decreases with rising GB thickness (Figure 15(a)). For a GB thickness $>5 \mathrm{~nm}$ the anisotropic $y$-GB $\left(H_{\text {ext }} \|[001]\right)$ has a lower $H_{\mathrm{sw}}$ compared to the isotropic $x y$-GB $\left(H_{\text {ext }} \|[111]\right)$. This is an explanation for the trend of higher $H_{\mathrm{sw}}$ values of magnets with higher misorientation degree, which contradicts the results formulated by Stoner and Wohlfarth [26] for noninteracting grains or particles but agrees with experimental results [52] and previous simulations [11]. In comparison, the dependence of the switching field of a $2-G$ model structure with averaged homogeneous magnetic properties in the GB layer $J_{s}=0.43 \mathrm{~T}$ and $A=1.00 \mathrm{pJ} / \mathrm{m}$ and $J_{s}=1.1 \mathrm{~T}$ and $A=6.54 \mathrm{pJ} / \mathrm{m}$, respectively, is shown in Figure 15(a) (dotted lines). With a low $J_{s}$ value $(0.43 \mathrm{~T})$ of the GB layer and $H_{\text {ext }} \|$ [111] $H_{\text {sw }}$ is above the value of the anisotropic $y$-GB $\left(H_{\text {ext }} \|[001]\right)$. The switching field value of the averaged GB $\left(H_{\text {ext }} \|[111]\right)$ with a $J_{s}$ of $1.10 \mathrm{~T}$ is below $H_{\mathrm{sw}}$ of the $y$-GB $\left(H_{\text {ext }} \|\right.$ [001]) for all GB thicknesses. At a GB thickness of about $4 \mathrm{~nm}$ the $x y$-GB and the homogeneous GB with a $J_{s}$ of $0.43 \mathrm{~T}$ have approximately the same switching field values. Therefore it is justified to use a single phased GB with homogeneous 

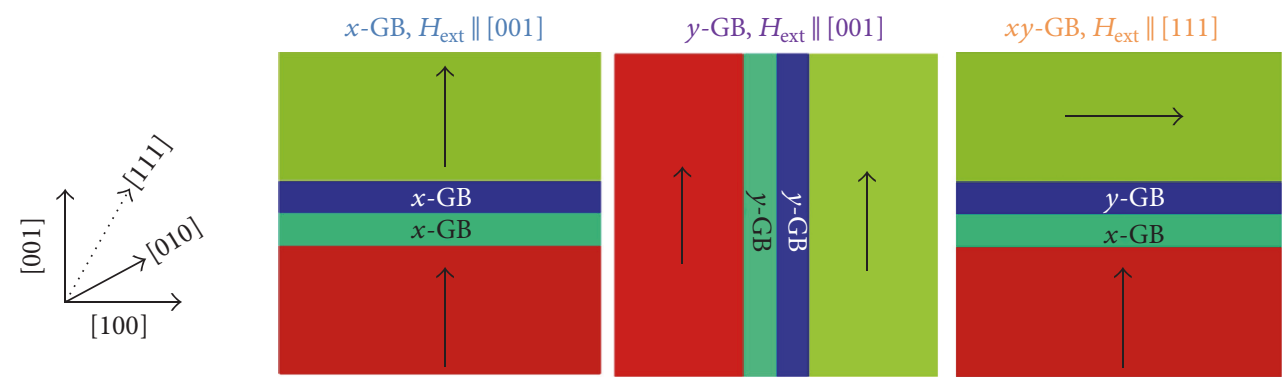

FIGURE 14: Three different configurations with the orientation of the GB parallel and normal to $H_{\text {ext }}$ and the $c$-axis of the grain perpendicular to the GB $(x-\mathrm{GB})$ and parallel to the GB $(y-\mathrm{GB})$.

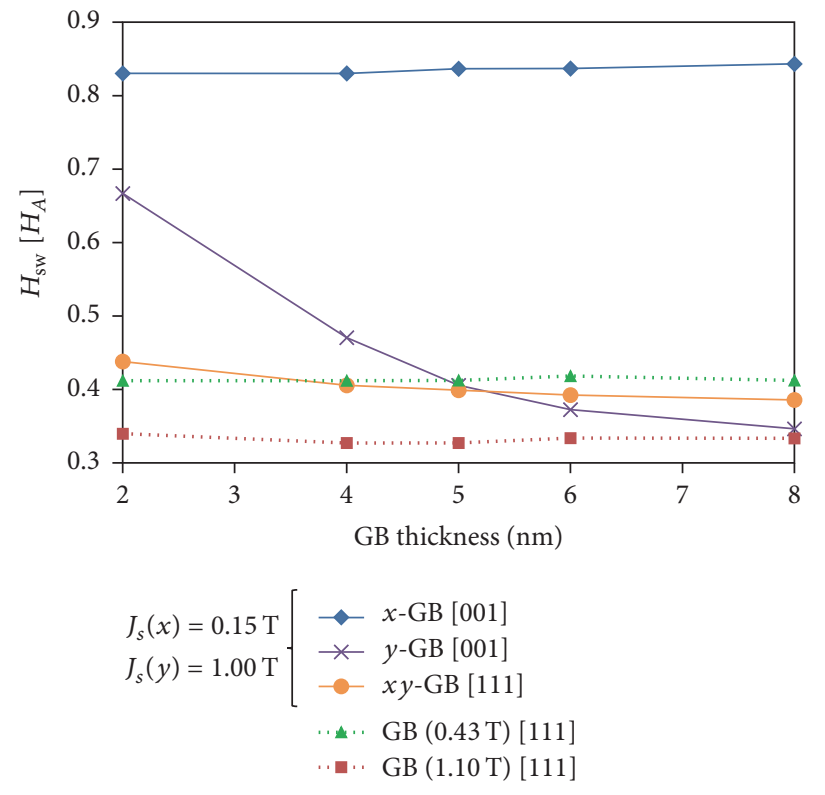

(a)

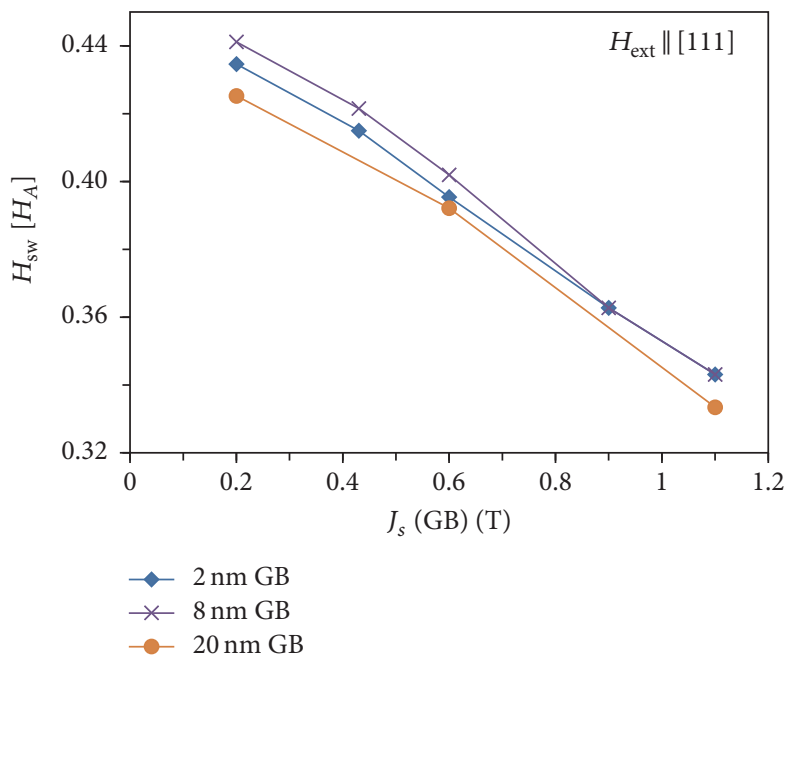

(b)

FIGURE 15: (a) Influence of the GB thickness on $H_{\mathrm{sw}}$ : for the three different 2-G model structures of Figure 14 (solid lines). In comparison the GB with averaged homogeneous magnetic properties of $J_{s}=0.43 \mathrm{~T} J_{s}=1.10 \mathrm{~T}$ are shown (dotted line). (b) Influence of the averaged homogeneous saturation polarization of the GB-phase on $H_{\text {sw }}$ in the 2-G model structure for different GB thickness, $H_{\text {ext }} \|$ [111]. The 2-G model structure with a GB thickness of $20 \mathrm{~nm}$ has a size of $60 \times 60 \times 60 \mathrm{~nm}$.

magnetic properties in isotropic multigrain finite element model structures. Figure 15(b) shows that the switching field constantly decreases from $0.44 H_{A}$ to $0.33 H_{A}$ for $\mathrm{GB}$ thickness ranging from $2 \mathrm{~nm}$ to $20 \mathrm{~nm}$ with rising $J_{s}$ value of the GB layer, as shown in the results of the simulations of the 2-G model structure with an averaged homogeneous GB composition $\left(H_{\text {ext }} \|[111]\right)$. It should be noted that the switching field values of the isotropic cases $x y$-GB [111], GB $(0.43 \mathrm{~T})$ [111], and GB(1.10 T) [111] (Figure 15(a)) and 2, 8, and $20 \mathrm{~nm} \mathrm{~GB}$ (Figure 15(b)) are more or less independent of the GB layer thickness.

During the magnetization reversal processes different types of domain wall (DW) types, such as Bloch and Néel DWs, are formed in perfectly aligned magnets depending on the orientation of the GB with respect to the $c$-axis of the adjacent grains and the direction of the external field. The calculated demagnetization curves for the pure $x$-GB with $H_{\text {ext }} / /[001]$ and $J_{s}=0.15 \mathrm{~T}$ (Table 3 ) and for the pure $y$-GB with $H_{\text {ext }} / /[001]$ and $J_{s}=1.0 \mathrm{~T}$ and a GB thickness of $8 \mathrm{~nm}$ are shown in Figure 16. As a result of the large difference in $J_{s}$ and $A$ values the coercive field for $x$ - and $y$-GB varies from $2.7 \mathrm{~T}$ to $6.5 \mathrm{~T}$. The $x$-GB shows a $12 \%$ higher coercive field, if the magnetic properties of $x$ - and $y$-GB are the same. This difference is originated by the different total energies for the formation of a Bloch domain wall (DW) $(y-\mathrm{GB})$ and a Néel DW $(x-\mathrm{GB})$ with an additional stray field contribution.

The magnetization of the $x$-GB rotates in the perpendicular direction with respect to the adjacent grains at a relatively small external field of $0.95 \mathrm{~T}$ (Figure 17(1)). Two Néel DWs are formed, whereby the magnetization within the center of the 


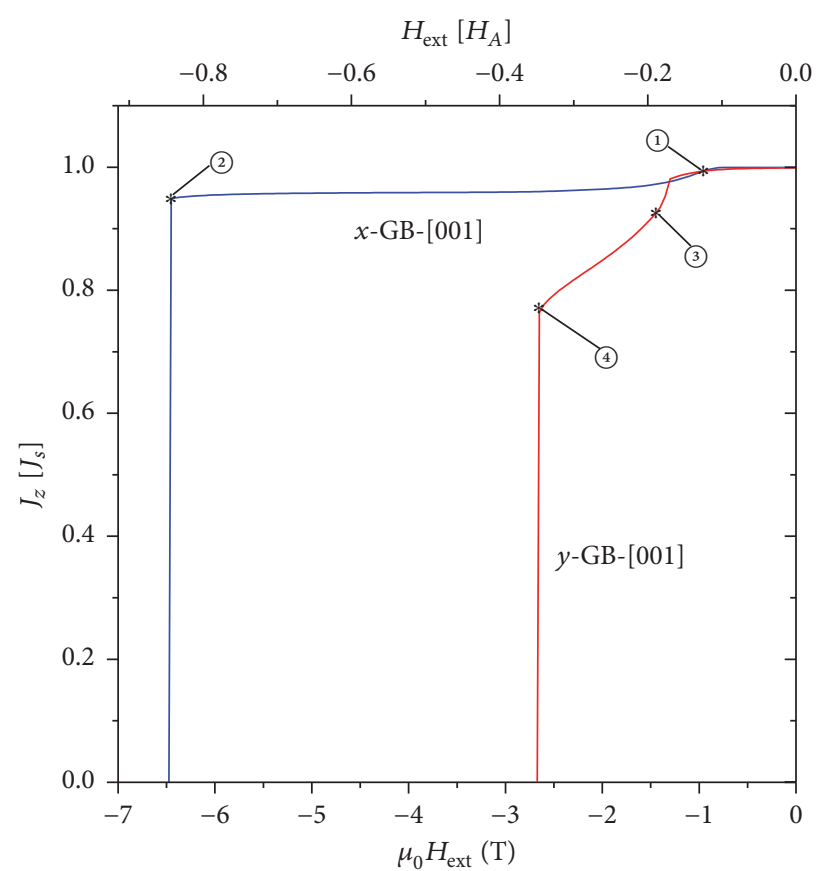

Figure 16: Calculated demagnetization curves for $x$-GB with $H_{\text {ext }} / /$ [001] and $y$-GB with $H_{\text {ext }} / /[001]$ and a GB thickness of $8 \mathrm{~nm}$. The details of the magnetic states (1)-(4) are shown in Figures 17 and 18.
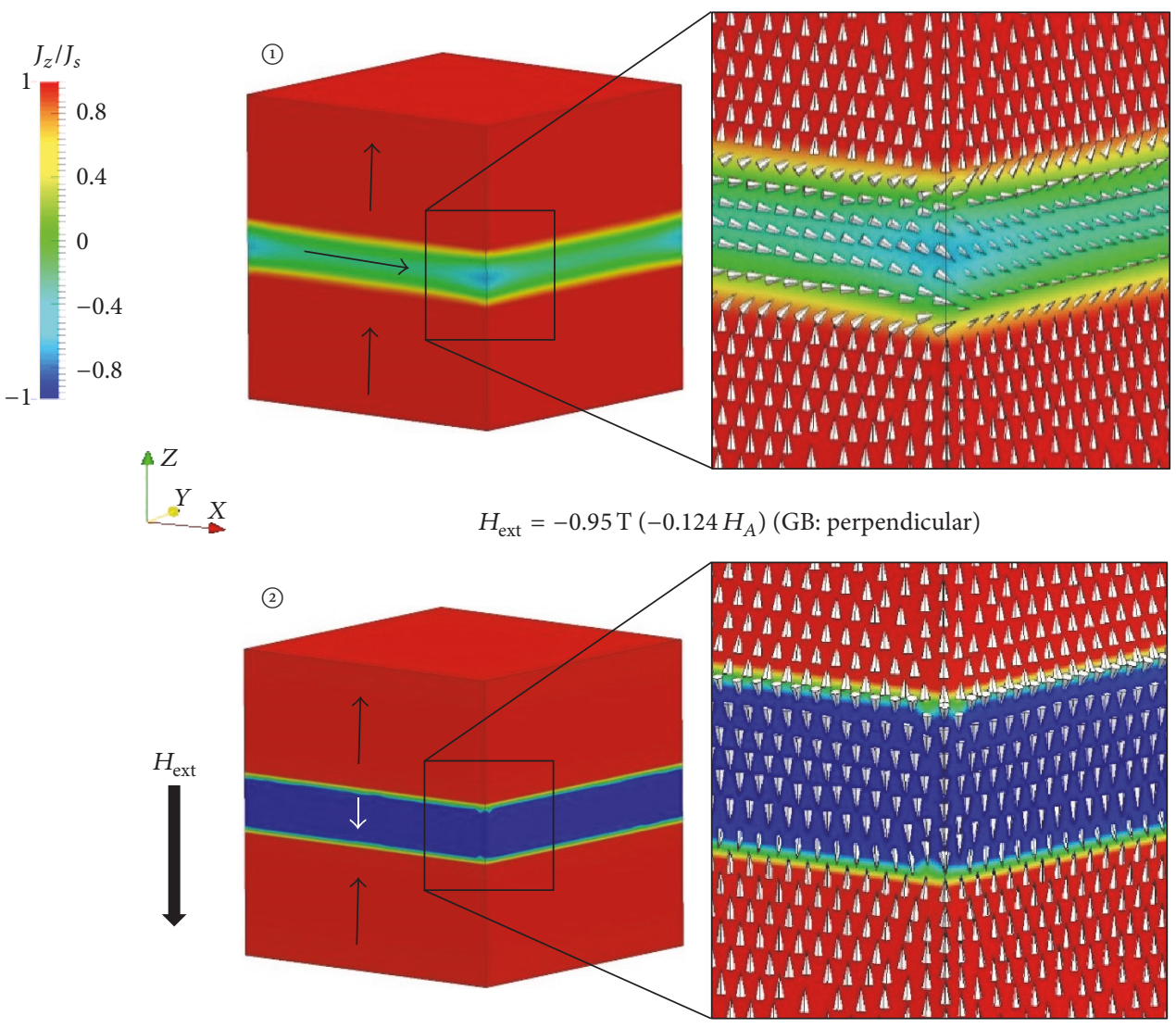

$H_{\text {ext }}=-6.45 \mathrm{~T}\left(-0.843 H_{A}\right)($ GB: antiparallel $)$

FIGURE 17: Calculated magnetization states of the $x$-GB with $H_{\text {ext }} / /$ [001]: (1) the magnetization of the GB is in plane and (2) the magnetization of the GB is parallel to the external field and antiparallel to the adjacent grains forming two Néel DWs close to the grain surfaces. 

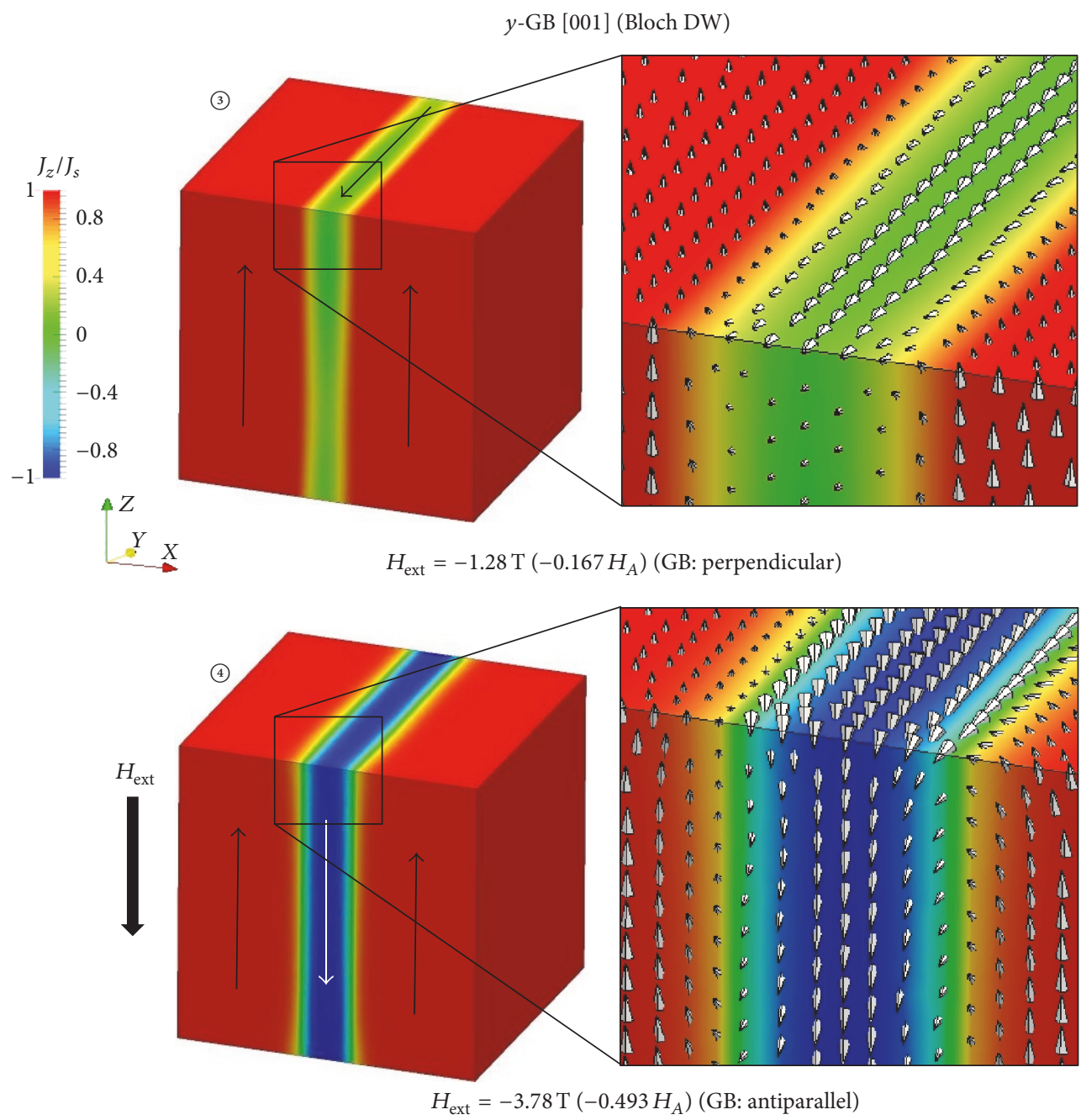

Figure 18: Magnetization states of the $y$-GB [001]: (3) the magnetization of the GB is perpendicular to $H_{\text {ext }}$ and the adjacent grains and (4) the magnetization of the GB is parallel to the external field and antiparallel to the adjacent grains forming a Bloch DW.

GB is antiparallel to one of the adjacent grains, until being at a high external field value of $6.45 \mathrm{~T}$ (Figure 17(2)). The high value of the necessary external field is originated by the large formation energy of a Néel DW due to the strong stray field occurring along the whole interfaces between the GB and the neighbouring grains.

The magnetization reversal state (3) is typical for a Bloch DW nucleated in the $y$-GB (Figure 183). Since the magnetization vector has a degree of freedom to rotate along the $y$-axis with relatively low activation energy, the $y$ GB switches at a lower external field of $3.78 \mathrm{~T}$ and finally forms two Bloch DWs at the interfaces with the hard magnetic grains (Figure 18(4)). The formation energy of the stray-field-free Bloch DWs is smaller than the one of the Néel DWs. In general the DWs are complex magnetization transitions between neighbouring magnetic domains. Their energy, thickness, and shape depend on various parameters such as the intrinsic magnetic properties and the shape of the magnetic material. The complex structure of DWs can only be calculated numerically by means of micromagnetic simulations [53].

The saturation polarization and the thickness of the GB layer have been varied using the isotropic Voronoi model structure of Figure 1 in order to verify the results of the 2-G model structure of Figure 15 with a realistic model structure with averaged homogeneous magnetic properties. At a small value of $J_{s}$ and $A$ the GB magnetically decouples the isotropically orientated hard magnetic grains leading to an increase of $H_{c J}$ with respect to direct coupled $\mathrm{Nd}_{2} \mathrm{Fe}_{14} \mathrm{~B}$ grains (Figure 19(a)). This behaviour is strongly pronounced in the MQU-F magnet material and also present in the MQP$\mathrm{B}+$ ribbon. As $J_{s}$ and $A$ of the GB-phase rise, $H_{c J}$ decreases linearly due to stronger coupling of the hard magnetic grains and the higher probability of a nucleation of a reverse magnetic domain in the GB. Simultaneously the remanence increases because of the stronger remanence enhancement effect of the coupled Nd-Fe-B grains [54]. At a GB thickness of $5 \mathrm{~nm}$ and grain size of $50 \mathrm{~nm}$ the coercive fields for the 


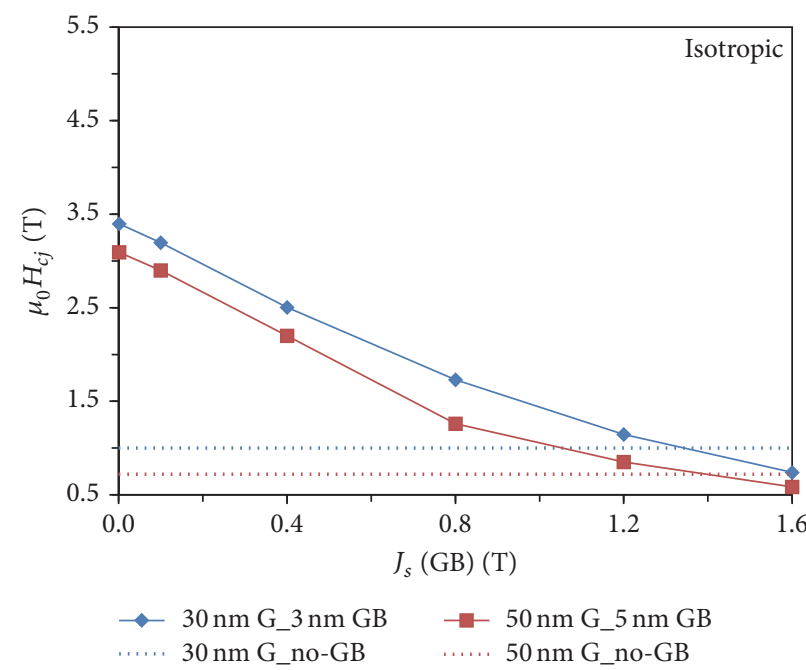

(a)

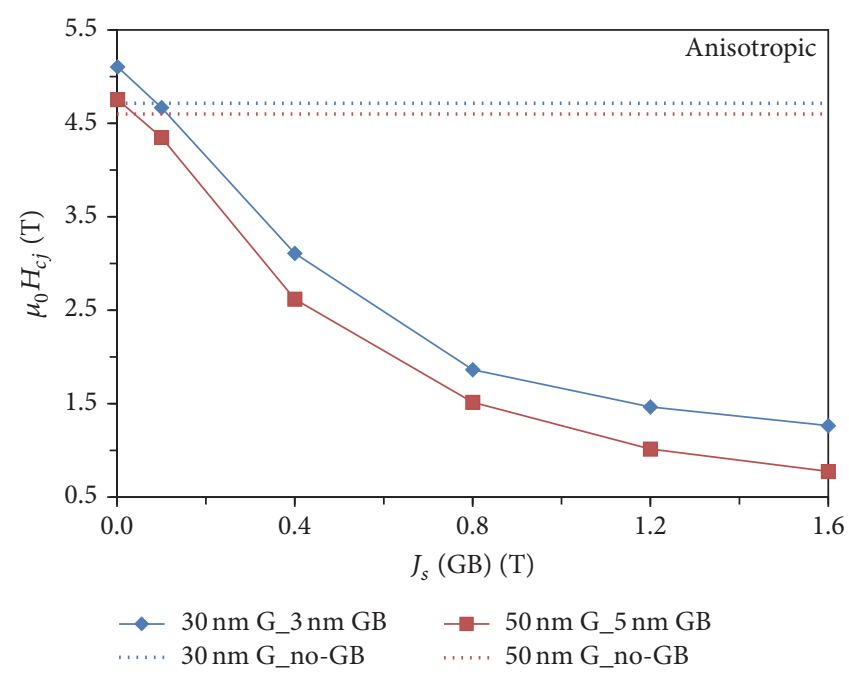

(b)

FIGURE 19: Influence of the averaged magnetic properties, the grain size and GB thickness on the coercive field. (a) Isotropically oriented grains. (b) Isotropically oriented grains.

model structures with and without a GB-phase are equal at $J_{s} \approx 1.40 \mathrm{~T}(A=10.60 \mathrm{pJ} / \mathrm{m})$ and equal at $J_{s} \approx 1.34 \mathrm{~T}$ $(A=9.71 \mathrm{pJ} / \mathrm{m})$ for a GB thickness of $3 \mathrm{~nm}$ and a grain size of $30 \mathrm{~nm}$ (Figure 19(a)). The further increase in $J_{s}$ and $A$ leads to a reduction of $H_{c J}$ with respect to directly coupled $\mathrm{Nd}_{2} \mathrm{Fe}_{14} \mathrm{~B}$ grains. In these simulations the ratio between the grain size and the GB thickness was kept constant. This accredits the significant difference in $H_{c J}$ of the $30 \mathrm{~nm} \mathrm{G}_{-} 3 \mathrm{~nm} \mathrm{~GB}$ and $50 \mathrm{~nm}$ G_ $5 \mathrm{~nm}$ GB simulations. This influence of the grain size is approximately equal to the difference of the calculated $H_{c j}$ values of the simulations of the model structure of directly coupled grains without a GB-phase (dotted lines in Figure 19(a)). Bance et al. [55] showed that the decrease of $H_{c J}$ with increasing grain size in hard magnets is caused by the nonuniform magnetostatic field in the polyhedral grains. In summary the results from the $2-G$ model structure that $H_{c j}$ is mostly independent of the GB thickness in isotropically oriented $\mathrm{Nd}-\mathrm{Fe}-\mathrm{B}$ magnets were also verified with the realistic Voronoi model structure calculations.

The dependence of $H_{c J}$ on the GB properties is more strongly pronounced in aligned Nd-Fe-B magnets. Figure 19(b) compares the results of simulations using the Voronoi model structure of Figure 1 with an average grain misalignment $\left\langle\vartheta_{0}\right\rangle \approx 7^{\circ}$. We observed that the decrease of $H_{c J}$ with rising grain size is less pronounced in the simulations of anisotropically oriented directly coupled Nd-Fe-B grains (dotted lines in Figure 19(b)). Secondly, the GB thickness has a stronger influence on the reduction of $H_{c J}$ in anisotropic magnets, which is shown in the greater difference in the $H_{c J}$ values of the $30 \mathrm{~nm}$ G_3 nm GB and $50 \mathrm{~nm} \mathrm{G \_ 5} \mathrm{nm} \mathrm{GB} \mathrm{simulations}$ compared to the directly coupled simulations (no-GB). This is in accordance with our recently published results of the strong decrease of $H_{c J}$ with rising GB thickness in anisotropic $\mathrm{Nd}-\mathrm{Fe}-\mathrm{B}$ magnets [11]. It should be emphasized that the presence of a soft magnetic GB layer always leads to a reduction of the coercive field in aligned magnet, if the saturation polarization of the GB is $>0.1 \mathrm{~T}(A=0.05 \mathrm{pJ} / \mathrm{m})$. The decrease of $H_{c I}$ with rising $J_{s}$ of the GB layer shows a nonlinear behaviour in anisotropically oriented grains, compared to the linear decrease in the isotropic case.

\section{Conclusion}

The TEM/EELS analysis of nanocrystalline Nd-Fe-B based magnet materials revealed an asymmetric composition profile of the $\mathrm{Fe}$ - and the Nd-content across the grain boundary phase in isotropically oriented melt-spun magnets. We found an enrichment of iron up to 60 at $\%$ in the $\mathrm{Nd}$-containing grain boundaries close to the prismatic $\mathrm{Nd}_{2} \mathrm{Fe}_{14} \mathrm{~B}$ grain surfaces and a reduced iron content up to $35 \%$ close to basal grain surfaces perpendicular to the $c$-axis. Numerical micromagnetic simulations based on granular Voronoi model structures showed that the coercive field strongly depends on the average $\mathrm{Fe}$-content and the saturation polarization and exchange stiffness constant of the GB-phase as well as on the GB thickness and grain orientation. In general, the coercive field is significantly increased, if the Fe-content of the GBs, especially parallel to the $c$-direction of the hard magnetic 2 14-1 grains, is reduced. Our simulations predicted an increase of the coercive field of isotropically oriented magnets with a soft magnetic GB-phase independently of the grain boundary thickness between $2 \mathrm{~nm}$ and $20 \mathrm{~nm}$ for $\left\langle J_{s}\right\rangle<1.2 \mathrm{~T}$ compared to directly coupled 2-14-1 grains with no-GB-phase. Contrary to this result we have demonstrated that the coercive field of anisotropic, aligned magnets significantly decreases for soft magnetic GB-phases with $J_{s}>0.2 \mathrm{~T}$ and GB thickness of $3 \mathrm{~nm}-5 \mathrm{~nm}$ compared to directly coupled 2-14-1 grains. Moreover a rising GB thickness $>4 \mathrm{~nm}$ further leads to a significant reduction in coercive field in anisotropic aligned magnets.

We have demonstrated that numerical micromagnetic simulations perfectly predict the hysteretic properties of 
$\mathrm{Nd}_{2} \mathrm{Fe}_{14} \mathrm{~B}$ based magnets compared to measured properties, if their microstructure has been quantitatively analyzed by TEM/EELS investigations.

\section{Conflicts of Interest}

The authors declare that there are no conflicts of interest regarding the publication of this paper.

\section{Acknowledgments}

The authors are grateful to Dr. P. Toson for helpful discussions and Dr. D. Brown from Magnequench Technology Center Singapore, for providing the rapidly quenched NdFe-B magnet samples. The TEM/STEM investigations were carried out using facilities at the University Service Center for Transmission Electron Microscopy, Vienna University of Technology, Austria. The authors acknowledge the TU Wien University Library for financial support through its Open Access Funding Programme.

\section{References}

[1] J. Fidler, T. Schrefl, S. Hoefinger, and M. Hajduga, "Recent developments in hard magnetic bulk materials," Journal of Physics Condensed Matter, vol. 16, no. 5, pp. S455-S470, 2004.

[2] J. Fidler and K. Knoch, "Electron microscopy of Nd-Fe-B based magnets," Journal of Magnetism and Magnetic Materials, vol. 80, no. 1, pp. 48-56, 1989.

[3] J. Fidler, J. Bernardi, and T. Schrefl, "Permanent magnets-new microstructural aspects," Scripta Metallurgica et Materialia, vol. 33, no. 10-11, pp. 1781-1791, 1995.

[4] J. Fidler, K. G. Knoch, H. Kronmüller, and G. Schneider, "Analytical TEM study of Al-doped, 'two-phase' Nd-Fe-B sintered magnets," Journal of Materials Research, vol. 4, no. 4, pp. 806814, 1989.

[5] K. G. Knoch, G. Schneider, J. Fidler, E.-T. Henig, and H. Kronmüller, "Al-doped Nd-Fe-B permanent magnets: wetting and microstructural investigations," IEEE Transactions on Magnetics, vol. 25, no. 5, pp. 3426-3428, 1989.

[6] J. Bernardi, J. Fidler, M. Seeger, and H. Kronmuller, "Preparation and TEM-study of sintered Nd18Fe74B6GalNb1 magnets," IEEE Transactions on Magnetics, vol. 29, no. 6, pp. 2773-2775, 1993.

[7] J. Fidler, "Analytical microscope studies of sintered Nd-Fe-B magnets," IEEE Transactions on Magnetics, vol. 21, no. 5, pp. 1955-1957, 1985.

[8] J. Fidler and J. Bernardi, "Transmission electron microscope characterization of cast and hot-worked R-Fe-B:Cu(R=Nd,Pr) permanent magnets," Journal of Applied Physics, vol. 70, no. 10, pp. 6456-6458, 1991.

[9] Y. Toga, H. Moriya, H. Tsuchiura, and A. Sakuma, "First principles study on interfacial electronic structures in exchangespring magnets," Journal of Physics: Conference Series, vol. 266, no. 1, Article ID 012046, 2011.

[10] T. Schrefl and J. Fidler, "Finite element modeling of nanocomposite magnets," IEEE Transactions on Magnetics, vol. 35, no. 5, pp. 3223-3228, 1999.

[11] G. A. Zickler, J. Fidler, J. Bernardi, T. Schrefl, and A. Asali, "A combined TEM/STEM and micromagnetic study of the anisotropic nature of grain boundaries and coercivity in Nd-FeB magnets," Advances in Materials Science and Engineering, vol. 2017, Article ID 6412042, 12 pages, 2017.

[12] J. F. Herbst, " $\mathrm{R}_{2} \mathrm{Fe}_{14} \mathrm{~B}$ materials: intrinsic properties and technological aspects," Reviews of Modern Physics, vol. 63, no. 4, pp. 819-898, 1991.

[13] D. Brown, B.-M. Ma, and Z. Chen, "Developments in the processing and properties of NdFeb-type permanent magnets," Journal of Magnetism and Magnetic Materials, vol. 248, no. 3, pp. 432-440, 2002.

[14] D. N. Brown, Z. Wu, F. He, D. J. Miller, and J. W. Herchenroeder, "Dysprosium-free melt-spun permanent magnets," Journal of Physics: Condensed Matter, vol. 26, no. 6, p. 064202, 2014.

[15] A. Kirchner, J. Thomas, O. Gutfleisch, D. Hinz, K.-H. Müller, and L. Schultz, "HRTEM studies of grain boundaries in dieupset Nd-Fe-Co-Ga-B magnets," Journal of Alloys and Compounds, vol. 365, no. 1-2, pp. 286-290, 2004.

[16] H. Kim, Y. Kim, W. Jeon, and H. Kim, "Magnetic properties and microstructure of nanocrystalline $\mathrm{NdFeB}$ magnets fabricated by a modified hot working process," Journal of Magnetics, vol. 7, no. 4, pp. 138-142, 2002.

[17] R. F. Egerton, "The range of validity of EELS microanalysis formulae," Ultramicroscopy, vol. 6, no. 1, pp. 297-300, 1981.

[18] P. Mcguiness, O. Akdogan, A. Asali et al., "Replacement and Original Magnet Engineering Options (ROMEOs): A European Seventh framework project to develop advanced permanent magnets without, or with reduced use of, critical raw materials," JOM, vol. 67, no. 6, pp. 1306-1317, 2015.

[19] R. F. Egerton, Electron Energy-Loss Spectroscopy in the Electron Microscope, Plenum Press, New York, NY, USA, 2nd edition, 1996.

[20] T. Schrefl, D. Suess, W. Scholz, H. Forster, V. Tsiantos, and J. Fidler, "Finite element micromagnetics," in Computational Electromagnetics, P. Monk, C. Carstensen, S. Funken, W. Hackbusch, and R. H. W. Hoppe, Eds., pp. 165-181, Springer, Berlin, Germany, 2003.

[21] T. L. Gilbert, "A phenomenological theory of damping in ferromagnetic materials," IEEE Transactions on Magnetics, vol. 40, no. 6, pp. 3443-3449, 2004.

[22] P. Toson, Multiscale modelling of advanced hard magnets [Ph.D. thesis], Technische Universität Wien, Vienna, Austria, 2015.

[23] C. H. Rycroft, "VORO++: a three-dimensional voronoi cell library in C++," Chaos, vol. 19, no. 4, Article ID 041111, 2009.

[24] A. Ribes and C. Caremoli, "Salomé platform component model for numerical simulation," in Proceedings of the 31st Annual International Computer Software and Applications Conference (COMPSAC '07), pp. 553-564, July 2007.

[25] R. W. McCallum, A. M. Kadin, G. B. Clemente, and J. E. Keem, "High performance isotropic permanent magnet based on $\mathrm{Nd}$ Fe-B," Journal of Applied Physics, vol. 61, no. 8, pp. 3577-3579, 1987.

[26] E. C. Stoner and E. P. Wohlfarth, "A mechanism of magnetic hysteresis in heterogeneous alloys," Philosophical Transactions of the Royal Society A: Mathematical, Physical and Engineering Sciences, vol. 240, no. 826, pp. 599-642, 1948.

[27] A. Melendo, A. Coll, M. Pasenau, E. Escolano, and A. Monros, 2016, http://www.gidhome.com/.

[28] K. Sohlberg, T. J. Pennycook, W. Zhou, and S. J. Pennycook, "Insights into the physical chemistry of materials from advances in HAADF-STEM," Physical Chemistry Chemical Physics, vol. 17, no. 6, pp. 3982-4006, 2015. 
[29] G. A. Zickler, P. Toson, A. Asali, and J. Fidler, "Nanoanalytical TEM studies and micromagnetic modelling of Nd-Fe-B magnets," Physics Procedia, vol. 75, pp. 1442-1449, 2015.

[30] W. Tang, S. Zhou, R. Wang, and C. D. Graham, "An investigation of the Nd-rich phases in the Nd-Fe-B system," Journal of Applied Physics, vol. 64, no. 10, pp. 5516-5518, 1988.

[31] D. Lemarchand, P. Vigier, and B. Labulle, "On the oxygen stabilized Nd-rich phase in the Nd-Fe-B (-0) permanent magnet system," IEEE Transactions on Magnetics, vol. 26, no. 5, pp. 2649-2651, 1990.

[32] S. C. Wang and Y. Li, "In situ TEM study of Nd-rich phase in $\mathrm{NdFeB}$ magnet," Journal of Magnetism and Magnetic Materials, vol. 285, no. 1-2, pp. 177-182, 2005.

[33] T. T. Sasaki, T. Ohkubo, Y. Une, H. Kubo, M. Sagawa, and K. Hono, "Effect of carbon on the coercivity and microstructure in fine-grained Nd-Fe-B sintered magnet," Acta Materialia, vol. 84, pp. 506-514, 2015.

[34] W. F. Li, T. Ohkubo, and K. Hono, "Effect of post-sinter annealing on the coercivity and microstructure of Nd-Fe-B permanent magnets," Acta Materialia, vol. 57, no. 5, pp. 13371346, 2009.

[35] H. Sepehri-Amin, T. Ohkubo, T. Shima, and K. Hono, "Grain boundary and interface chemistry of an Nd-Fe-B-based sintered magnet," Acta Materialia, vol. 60, no. 3, pp. 819-830, 2012.

[36] T. G. Woodcock, Q. M. Ramasse, G. Hrkac et al., "Atomicscale features of phase boundaries in hot deformed Nd-Fe-CoB-Ga magnets infiltrated with a Nd-Cu eutectic liquid," Acta Materialia, vol. 77, pp. 111-124, 2014.

[37] T. T. Sasaki, T. Ohkubo, and K. Hono, "Structure and chemical compositions of the grain boundary phase in Nd-Fe-B sintered magnets," Acta Materialia, vol. 115, pp. 269-277, 2016.

[38] T.-H. Kim, S.-R. Lee, M.-W. Lee et al., "Dependence of magnetic, phase-transformation and microstructural characteristics on the $\mathrm{Cu}$ content of Nd-Fe-B sintered magnet," Acta Materialia, vol. 66, pp. 12-21, 2014.

[39] H. Sepehri-Amin, W. F. Li, T. Ohkubo, T. Nishiuchi, S. Hirosawa, and K. Hono, "Effect of Ga addition on the microstructure and magnetic properties of hydrogenation-disproportionationdesorption-recombination processed Nd-Fe-B powder," Acta Materialia, vol. 58, no. 4, pp. 1309-1316, 2010.

[40] A. Sakuma, T. Suzuki, T. Furuuchi, T. Shima, and K. Hono, "Magnetism of Nd-Fe films as a model of grain boundary phase in Nd-Fe-B permanent magnets," Applied Physics Express, vol. 9, no. 1, Article ID 13002, 2016.

[41] H. Kronmüller and M. Fähnle, Micromagnetism and the Microstructure of Ferromagnetic Solids, Cambridge University Press, New York, NY, USA, 2003.

[42] J. A. Horton, M. K. Miller, L. Heatherly, J. W. Jones, K. F. Russel, and V. Panchanathan, "Microstructural characterization of high energy product Nd-Fe-B rapidly solidified ribbons," ORNLCP98593, 1998, https://inis.iaea.org/search/search.aspx?orig_q= $\mathrm{RN}: 30013898$.

[43] M. Shiga and H. P. J. Wijn, "Figs. 30-51, Tables 5-9: datasheet from Landolt-Börnstein-group III condensed matter," in $3 d, 4 d$ and 5 d Elements, Alloys and Compounds, vol. 19 of Magnetic Properties of Metals, p. 536, Springer, Berlin, Germany, 1986, http:// materials.springer.com/lb/docs/sm_lbs_978-3-540-39667-3_86.

[44] R. Hawig, Y. Kahn, and H. Wibbeke, "Magnetic, electrical properties and crystallization behavior of amorphous $\mathrm{Nb}_{x} \mathrm{Fe}_{76} \mathrm{~B}_{24-x}$ alloys," Zeitschrift fuer Metallkunde/Materials Research and Advanced Techniques, vol. 82, pp. 646-649, 1991.
[45] N. M. Saiden, T. Schrefl, H. A. Davies, and G. Hrkac, "Micromagnetic finite element simulation of nanocrystalline $\alpha$ $\mathrm{Fe} / \mathrm{Nd}_{2} \mathrm{Fe}_{14} \mathrm{~B} / \mathrm{Fe}_{3} \mathrm{~B}$ magnets," Journal of Magnetism and Magnetic Materials, vol. 365, pp. 45-50, 2014.

[46] J. F. Herbst and W. B. Yelon, "Preferential site occupation and magnetic structure of Nd 2(CoxFel-x) 14B systems," Journal of Applied Physics, vol. 60, no. 12, pp. 4224-4229, 1986.

[47] S. Ohba, Y. Saito, and Y. Noda, "A measurement of charge asphericity in iron metal," Acta Crystallographica Section A, vol. 38, no. 5, pp. 725-729, 1982.

[48] A. M. Van der Kraan and K. H. J. Buschow, "The ${ }^{57}$ Fe Mössbauer isomer shift in intermetallic compounds of iron," Physica $B+C$, vol. 138, no. 1-2, pp. 55-62, 1986.

[49] R. Skomski and J. M. D. Coey, Permanent Magnetism, Institute of Physics, Bristol, UK, 1999.

[50] R. Skomski, Simple Models of Magnetism, Oxford University Press, New York, NY, USA, 2008.

[51] E. Kneller, A. Seeger, and H. Kronmüller, "Ferromagnetismus," in Mit einem Beitrag Quantentheorie und Elektronentheorie des Ferromagnetismus, E. Kneller, A. Seeger, and H. Kronmüller, Eds., pp. 30-37, Springer, Berlin, Germany, 1962.

[52] K. Uestuener, M. Katter, and W. Rodewald, "Dependence of the mean grain size and coercivity of sintered Nd-Fe-B magnets on the initial powder particle size," IEEE Transactions on Magnetics, vol. 42, no. 10, pp. 2897-2899, 2006.

[53] D. Suess, J. Fidler, K. Porath, T. Schrefl, and D. Weller, "Micromagnetic study of pinning behavior in percolated media," Journal of Applied Physics, vol. 99, no. 8, Article ID 08G905, 2006.

[54] G. B. Clemente, J. E. Keem, and J. P. Bradley, “The microstructural and compositional influence upon HIREM behavior in Nd2Fe14B," Journal of Applied Physics, vol. 64, no. 10, pp. 52995301, 1988.

[55] S. Bance, B. Seebacher, T. Schrefl et al., "Grain-size dependent demagnetizing factors in permanent magnets," Journal of Applied Physics, vol. 116, no. 23, Article ID 233903, 2014. 

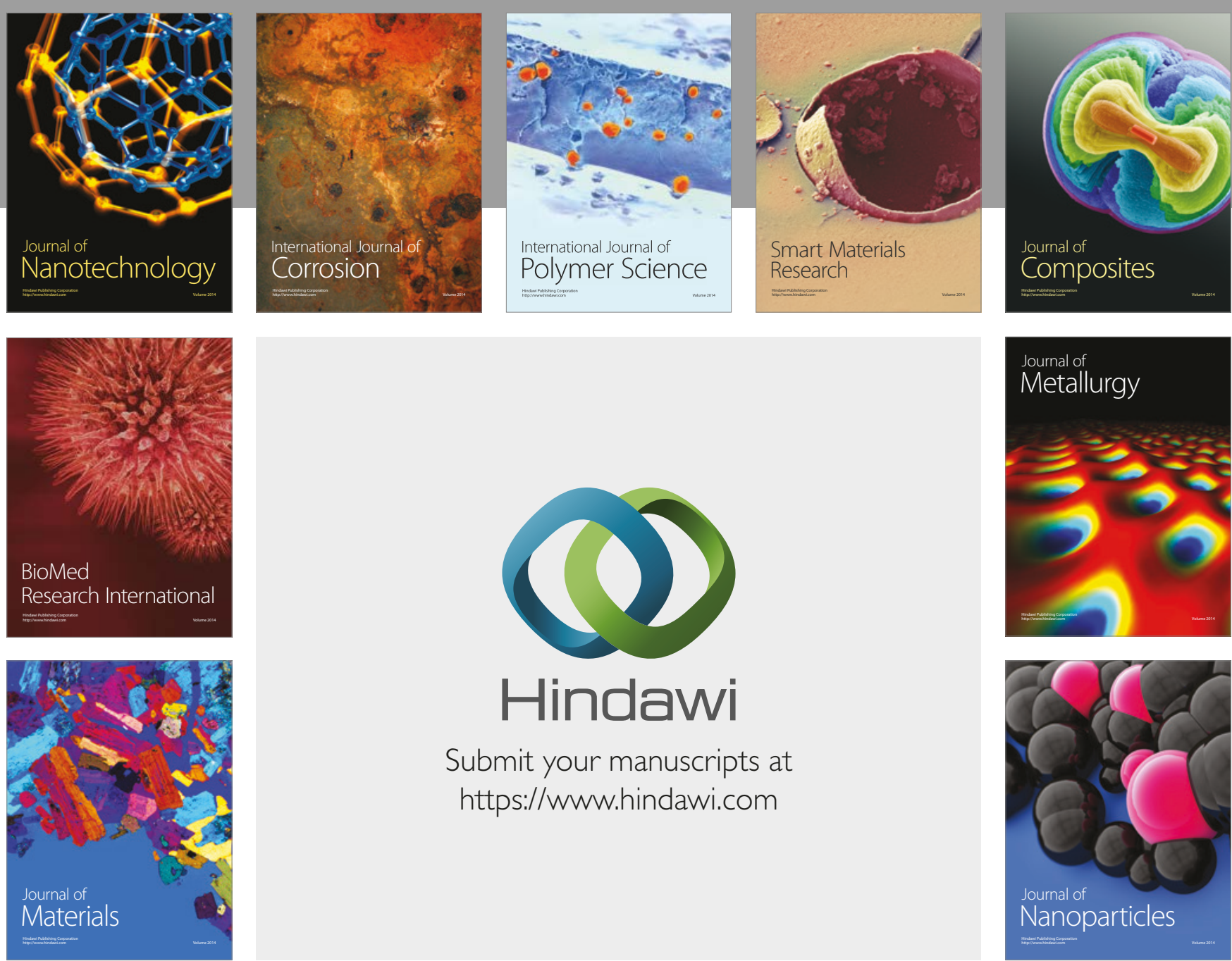

\section{Hindawi}

Submit your manuscripts at

https://www.hindawi.com
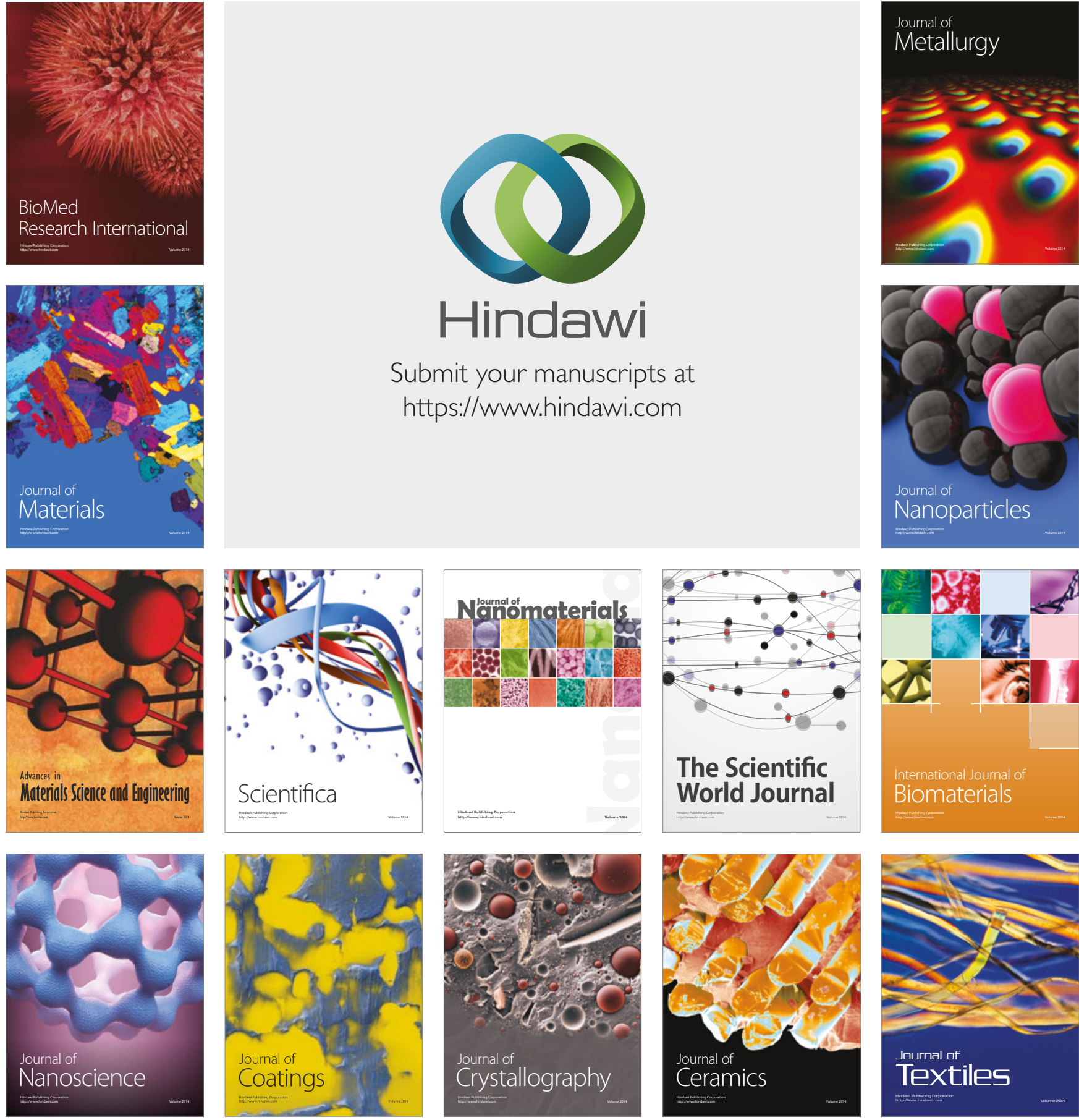

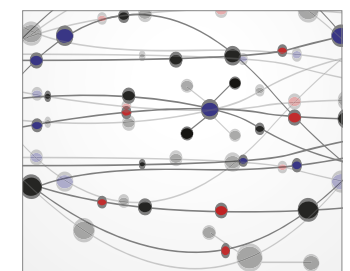

The Scientific World Journal
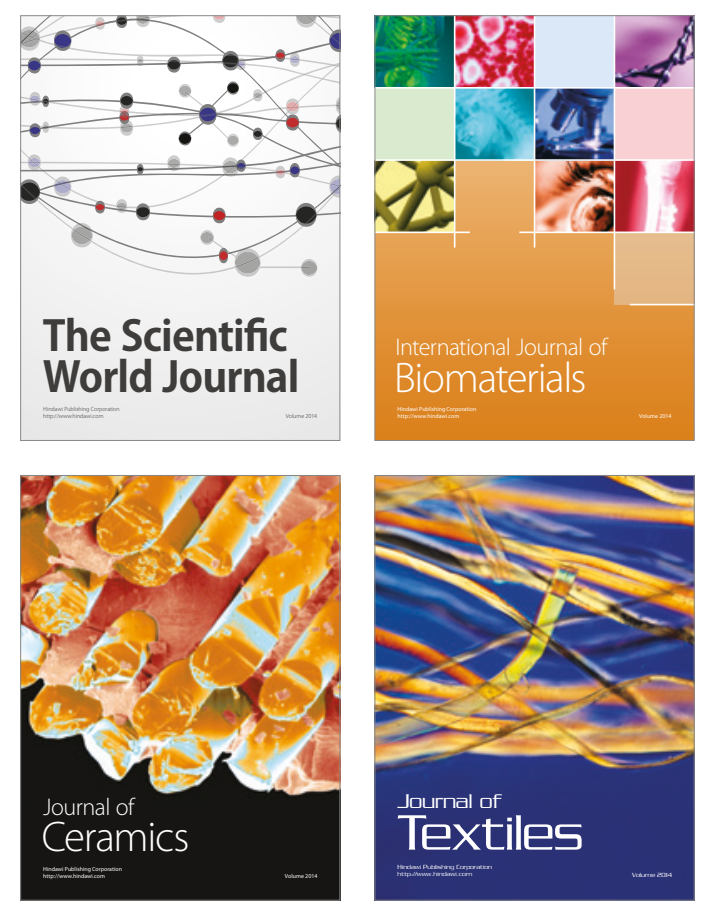